\title{
Momentum reconstruction and contact of the one-dimensional Bose-Fermi mixture
}

\author{
Ovidiu I. Pâţu ${ }^{1}$ and Andreas Klümper ${ }^{2}$ \\ ${ }^{1}$ Institute for Space Sciences, Bucharest-Măgurele, $R$ 077125, Romania \\ ${ }^{2}$ Fakultät für Mathematik und Naturwissenschaften, \\ Bergische Universität Wuppertal, 42097 Wuppertal, Germany
}

\begin{abstract}
We investigate the one-dimensional mixture of scalar bosons and spin polarized fermions interacting through a $\delta$-function potential. Using a thermodynamic description derived by employing a lattice embedding of the continuum model and the quantum transfer matrix method we perform a detailed analysis of the contact and quantum critical behaviour. We show that the compressibility Wilson ratio presents anomalous enhancement at the quantum critical points and that the boundaries of the quantum critical regions can be well mapped by the maxima of the specific heat. As a function of the coupling strength and temperature the contact presents nonmonotonic behavior. In the strong coupling regime the local minimum exhibited by the contact as a function of temperature is accompanied by a significant momentum reconstruction at both low and high momenta. This momentum reconstruction occurs as the system crosses the boundary between the Tomonaga-Luttinger liquid phase to the spin-incoherent regime and provides an experimental signature of the transition.
\end{abstract}

\section{INTRODUCTION}

Physical systems of ultracold atomic gases are characterized by a high degree of control over interaction strength, statistics and dimensionality which makes them ideal candidates for the investigation of various quantum many-body phenomena [1]3. The absence of defects and impurities makes these systems particularly suited for the simulation of many condensed matter models but at the same time they also allow for the creation of more exotic quantum systems. One example is the degenerate mixture of bosons and fermions which has been experimentally realized in various trap and lattice geometries. The study of Bose-Fermi mixtures (BFM) is extremely important from the experimental point of view due to the sympathetic cooling of fermions via interactions with bosons [4] but also theoretically because they exhibit phases and phenomena which are seldom studied in the condensed matter context. One-dimensional BFM, which are characterized by enhanced quantum fluctuations, have been investigated - both on the lattice and the continuum using mean-field theory [5] 8 , bosonization (TomonagaLuttinger liquid) 9 15, density waves [16, 17, exact solutions 18 32, and various numerical approaches 3341. The phase diagram is very rich and contains Mott insulators, spin and charge density waves, phase separation, Tomonaga-Luttinger and spin-incoherent liquids and Wigner crystals. In recent years there have also been an increasing number of studies on few-body mixtures, which are, in general, focused on the strong coupling regime. Various methods are employed such as: the multi-component generalization of the Bose-Fermi mapping [42 51], approximation by spin-chains [52 54, energy-functional techniques $55+58$ and trial wave functions 59 61.

In this article we study the one-dimensional (1D) mixture of scalar bosons and spin polarized fermions with contact interactions in the continuum. This system has been investigated in several papers but the vast majority of them were restricted to the study of the ground state.
However, experiments are performed at finite temperature which highlights the need for the computation of accurate thermodynamic data. For example, many multicomponent systems present quantum phase transitions (QPTs) at zero temperature 62] as certain parameters are varied (pressure, magnetic field, doping, etc.). The effects of these QPTs can also be detected at finite temperature in the so-called quantum critical (QC) region which is characterized by strong coupling of the thermal and quantum fluctuations. While the zero temperature phase diagram gives the quantum critical points the determination of the boundaries of the $\mathrm{QC}$ regions can be done only by computing the thermodynamic properties.

The 1D BFM with contact interactions is integrable when the masses of the fermions and bosons and all the coupling strengths are independently equal [18, 24] 26. In this case powerful methods associated with Bethe Ansatz 63, 64 can be employed to calculate various zero and finite temperature properties. In particular the thermodynamics of the system can be derived using the thermodynamic Bethe ansatz (TBA) 65, 66. In general, thermodynamic descriptions of integrable models derived using the TBA are characterized by an infinite number of integral equations 66 which makes their numerical implementation very difficult. While the BFM is one of the very few exceptions from this rule 27] the TBA thermodynamics of a large number of integrable multi-component systems like the two-component Fermi (2CFG) [67, 68, or Bose gas (2CBG) 67] suffer from the same drawback. Other notable exceptions are systems characterized by $q$-deformed algebras at special roots of unity which quite typically leads to a truncation. One way of circumventing these difficulties is provided by the quantum transfer matrix (QTM) method 69 74] which has the advantage of producing a finite number of integral equations that are easier to implement numerically. In Refs. 75 77 the authors succeeded in deriving such thermodynamic descriptions for the 2CBG and 2CFG and in this article we show that the same method can also applied in the case of the Bose-Fermi mixture. 
Our result hints strongly that similar efficient thermodynamic descriptions involving only $\kappa$ integral equations for a $\kappa$-component system can be derived using the same method.

We use this result to perform a detailed analysis of the universal Tan contact [78 91] which governs the $1 / k^{4}$ of the momentum distribution. At finite temperature and as a function of the coupling strength the contact presents local maxima for small values of the boson fraction, a feature which is not present at zero temperature. Even more interesting, the contact develops a local minimum as a function of the temperature which results in a counterintuitive momentum reconstruction at the system's transition from the TLL phase to the incoherent regime. In addition, we determine the boundaries of the quantum critical regions which can be identified with the maxima of the grand canonical specific heat. Similar to the case of the $2 \mathrm{CBG}$ 92] the Wilson ratio presents anomalous enhancement in the vicinity of the quantum critical points and can be used to distinguish between different phases.

The plan of the paper is as follows. In Sec. II we introduce the model and in Sec. III we present the TBA thermodynamics and our results derived in the quantum transfer matrix framework. The analysis of the contact and momentum reconstruction is presented in Sec. IV and the determination of the boundaries of the QC regions is performed in Sec. V] The derivation of the thermodynamics is outlined in Secs. VI and VII. We conclude in Sec. VIII.

\section{THE MODEL}

The model investigated in this article describes onedimensional scalar bosons and spinless fermions with contact interactions. The Hamiltonian in second quantization is

$$
\begin{gathered}
H=\int d x \sum_{\sigma \in\{B, F\}}\left(\frac{\hbar^{2}}{2 m_{\sigma}} \partial_{x} \Psi_{\sigma}^{\dagger} \partial_{x} \Psi_{\sigma}-\mu_{\sigma} \Psi_{\sigma}^{\dagger} \Psi_{\sigma}\right) \\
+\frac{g_{B B}}{2} \Psi_{B}^{\dagger} \Psi_{B}^{\dagger} \Psi_{B} \Psi_{B}+g_{B F} \Psi_{B}^{\dagger} \Psi_{F}^{\dagger} \Psi_{F} \Psi_{B}
\end{gathered}
$$

where $\Psi_{B}(x)$ and $\Psi_{F}(x)$ are bosonic and fermionic fields satisfying canonical commutation and anticommutation relations, $m_{B}, m_{F}$ are the masses of the bosonic and fermionic particles, and $\mu_{B}$ and $\mu_{F}$ are the chemical potentials. In (1), $g_{B B}$ and $g_{B F}$ are the Bose-Bose and Bose-Fermi interaction strengths which can be expressed in terms of the $1 \mathrm{D}$ scattering lengths $a_{B B}$ and $a_{B F}$ via $g_{\sigma \sigma^{\prime}}=-\hbar^{2} / m_{\sigma \sigma^{\prime}} a_{\sigma \sigma^{\prime}}$ with $\sigma, \sigma^{\prime} \in\{B, F\}$ and $m_{\sigma \sigma^{\prime}}=\left(m_{\sigma}+m_{\sigma^{\prime}}\right) / m_{\sigma} m_{\sigma^{\prime}}$ the reduced mass.

The Hamiltonian (1) is integrable when the masses $m_{B}=m_{F}=m$ and coupling strengths are equal $g_{B B}=$ $g_{B F}=g[18,24,25$. This is the case that will be considered in the rest of this article and in order to make contact with the literature we are going to use units of $\hbar=2 m=1$ and introduce $g=2 c$ with $c>0$. For a system of $M$ particles of which $M_{B}$ are bosons and $M_{F}=M-M_{B}$ are fermions the energy spectrum of (1) is [24, 25]

$$
E_{B F}=\sum_{j=1}^{M}\left(k_{j}^{(1)}\right)^{2}-\mu_{B} M_{B}-\mu_{F}\left(M-M_{B}\right),
$$

with $\left\{k_{j}^{(1)}\right\}_{j=1}^{M}$ satisfying the Bethe ansatz equations (BAEs)

$$
\begin{aligned}
e^{i k_{s}^{(1)} L_{B F}} & =\prod_{p=1}^{M_{B}} \frac{k_{s}^{(1)}-k_{p}^{(2)}+i c / 2}{k_{s}^{(1)}-k_{p}^{(2)}-i c / 2}, \quad s=1, \cdots, M \\
1 & =\prod_{j=1}^{M} \frac{k_{l}^{(2)}-k_{j}^{(1)}+i c / 2}{k_{l}^{(2)}-k_{j}^{(1)}-i c / 2}, \quad l=1, \cdots, M_{B},
\end{aligned}
$$

where $L_{B F}$ is the length of the system and we have assumed periodic boundary conditions.

\section{THERMODYNAMICS}

\section{A. TBA result}

From the historical point of view the first method employed to determine the thermodynamics of an integrable model was the thermodynamic Bethe ansatz 66 introduced by Yang and Yang in their study of the LiebLiniger model [65]. In the TBA framework the BoseFermi mixture was investigated in Ref. [27. Introducing an effective magnetic field and chemical potential defined by $\mu=\left(\mu_{B}+\mu_{F}\right) / 2$ and $2 H=\mu_{B}-\mu_{F}$ the grand canonical potential per length is $(\beta=1 / T)$

$$
\phi_{Y C Z}(\mu, H, \beta)=-\frac{1}{2 \pi \beta} \int_{\mathbb{R}} \ln \left[1+e^{-\beta \epsilon(k)}\right] d k,
$$

with $\epsilon(k)$ satisfying the following system of non-linear integral equations (NLIEs)

$$
\begin{aligned}
& \epsilon(k)=k^{2}-\mu+H-\beta^{-1} \int_{\mathbb{R}} b_{1}(k-\lambda) \ln \left[1+e^{-\beta \varphi(\lambda)}\right] d \lambda, \\
& \varphi(\lambda)=-2 H-\beta^{-1} \int_{\mathbb{R}} b_{1}(\lambda-k) \ln \left[1+e^{-\beta \epsilon(k)}\right] d k
\end{aligned}
$$

with $b_{1}(k)=c /\left[2 \pi\left(c^{2} / 4+k^{2}\right)\right]$. It should be noted that in general the TBA description of multi-component systems involve an infinite number of NLIEs. Therefore, it is extremely fortunate that in the case of the BFM we encounter only two equations which is due to the fact that the Bethe equations (3) have only real solutions. However, in the case of all the other multi-component systems with contact interactions like the $2 \mathrm{CBG}$ and $2 \mathrm{CFG}$ and even a lot of single component systems the Bethe equations have complex solutions which means that the 


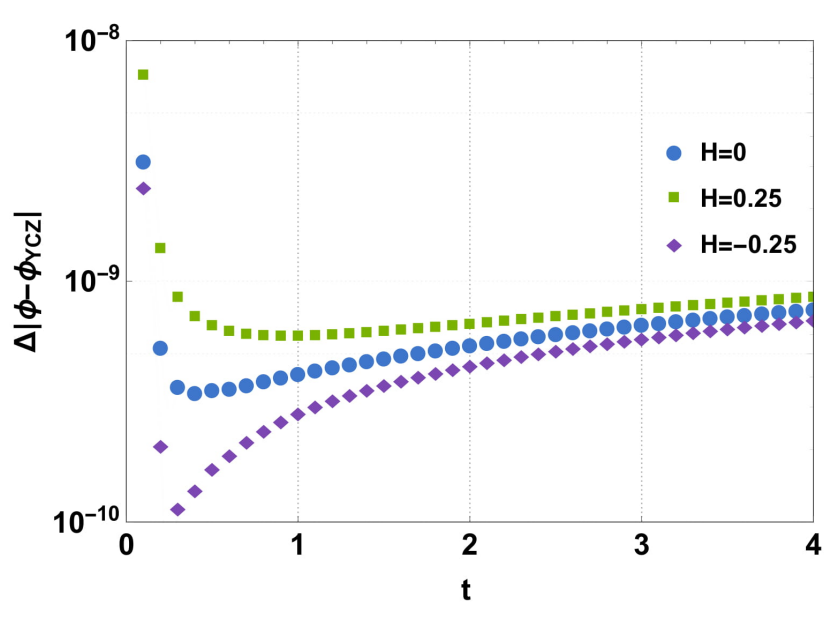

FIG. 1. Plot of the relative errors between the TBA grand canonical potential (4) and our result (6) for $c=1$ and $H=$ $-0.25,0,0.25$. Here $t=T / c^{2}$.

TBA description is very hard to implement numerically. A more efficient method which has the advantage of producing only a finite number of integral equations even for models whose BAEs admit complex solutions is the quantum transfer matrix (QTM) technique. Even though the QTM can be defined only for lattice models this difficulty can be circumvented by considering a lattice embedding for the continuum model. In Refs. [75] 77] the authors employed this method and succeeded in deriving a system of only two NLIEs characterizing the thermodynamics of the $2 \mathrm{CBG}$ and $2 \mathrm{CFG}$. The same method can be used in the case of the Bose-Fermi mixture as we will show below.

\section{B. Alternative thermodynamic description}

The lattice embedding of the BFM is the Perk-Schultz spin-chain with the $(-+-)$ grading (see Sec. VI). The derivation of the QTM thermodynamic description is relatively involved and will be presented in Sec. VII Here we present the main result and show the equivalence with the TBA description. The grand canonical potential per length is

$$
\phi(\mu, H, \beta)=-\frac{1}{2 \pi \beta} \int_{\mathbb{R}}\left[\ln A_{1}(k)+\ln A_{2}(k)\right] d k
$$

with the two auxiliary functions $a_{1,2}(k),\left(A_{1,2}(k)=1+\right.$ $\left.a_{1,2}(k)\right)$ satisfying the following system of NLIEs

$$
\begin{aligned}
\ln a_{1}(k)=-\beta & \left(k^{2}-\mu-H\right)+\int_{\mathbb{R}} K_{0}\left(k-k^{\prime}\right) \ln A_{1}\left(k^{\prime}\right) d k^{\prime} \\
& +\int_{\mathbb{R}+i \varepsilon} K_{2}\left(k-k^{\prime}\right) \ln A_{2}\left(k^{\prime}\right) d k^{\prime} \\
\ln a_{2}(k)=-\beta & \left(k^{2}-\mu+H\right) \\
& +\int_{\mathbb{R}-i \varepsilon} K_{1}\left(k-k^{\prime}\right) \ln A_{1}\left(k^{\prime}\right) d k^{\prime}
\end{aligned}
$$

where $\varepsilon \rightarrow 0$ and the kernels are defined by $K_{0}(k)=$ $\frac{1}{2 \pi} \frac{2 c}{k^{2}+c^{2}}, K_{1}(k)=\frac{1}{2 \pi} \frac{c}{k(k+i c)}$, and $K_{2}(k)=\frac{1}{2 \pi} \frac{c}{k(k-i c)}$.

We can analytically check the validity of our results in some particular cases. In the noninteracting limit, $c \rightarrow 0$, using $\lim _{c \rightarrow 0} K_{1}(k+i \varepsilon)=\lim _{c \rightarrow 0} K_{2}(k-i \varepsilon)=0$ and $\lim _{c \rightarrow 0} K_{2}(k)=\delta(k)$ the NLIEs (7) decouple

$$
\begin{aligned}
& \ln a_{1}(k)=-\beta\left(k^{2}-\mu-H\right)+\ln \left[1+a_{1}(k)\right], \\
& \ln a_{2}(k)=-\beta\left(k^{2}-\mu+H\right),
\end{aligned}
$$

and can be solved obtaining for the grand canonical potential $\phi(\mu, H, \beta)=\frac{1}{2 \pi \beta} \int_{\mathbb{R}} \ln \left[1-e^{-\beta\left(k^{2}-\mu-H\right)}\right] d k-$ $\frac{1}{2 \pi \beta} \int_{\mathbb{R}} \ln \left[1+e^{-\beta\left(k^{2}-\mu+H\right)}\right] d k$ which is the known result for a noninteracting mixture of fermions and bosons with different chemical potentials. For large values of $H$ the fermionic degrees of freedom are strongly suppressed, $a_{2}(k) \sim 0$. Eqs. (7) reduce to the Yang-Yang equation for the Lieb-Liniger model 65.

$\ln a_{1}(k)=-\beta\left(k^{2}-\mu-H\right)+\int_{\mathbb{R}} K_{0}\left(k-k^{\prime}\right) \ln A_{1}\left(k^{\prime}\right) d k^{\prime}$

and $\phi(\mu, H, \beta)=-\frac{1}{2 \pi \beta} \int_{\mathbb{R}} \ln \left[1+a_{1}(k)\right] d k$ which reproduces the TBA result for single component bosons with contact interactions. In the impenetrable limit $c \rightarrow \infty$ our result should coincide with the one obtained by Takahashi for two-component impenetrable fermions i.e.,

$\phi_{\infty}(\mu, H, \beta)=-\frac{1}{2 \pi \beta} \int_{\mathbb{R}} \ln \left[1+2 \cosh (\beta H) e^{-\beta\left(k^{2}-\mu\right)}\right] d k$.

While we have not succeeded in proving analytically the equivalence of our result with (8) we have checked it numerically and found perfect agreement.

The equivalence of the TBA and QTM thermodynamic descriptions is shown in Fig. 1 where we plot the numerically evaluated relative error defined as

$$
\Delta\left|\phi-\phi_{Y C Z}\right|=\frac{\left|\phi-\phi_{Y C Z}\right|}{\operatorname{Max}\left[\phi, \phi_{Y C Z}\right]},
$$

which shows that (4) and (6) (modulo numerical errors) produce identical results. Because in both cases we have $\phi(c, \mu, H, T)=c^{3} \phi\left(1, \mu / c^{2}, H / c^{2}, T / c^{2}\right)$ it is sufficient to consider only $c=1$. The computational complexities of both descriptions are the same which means that choosing one of them is a matter of personal choice. In the rest of the paper we use (6) and (7) mainly because our auxiliary functions have zero asymptotics at infinity resulting in a more precise treatment of convolutions using the Fast Fourier Transform.

The thermodynamic descriptions for the 2CBG 75 , 76, 2CFG [77 and BFM, (4) and (7), derived in the quantum transfer matrix framework involve only two auxiliary functions, $a_{1,2}(k)$, and the same expression for the grand canonical potential (6). The system of NLIEs is different in each case and can be compactly written as 


$$
\begin{aligned}
& \left([f * g](x)=\int_{\mathbb{R}} f\left(x-x^{\prime}\right) g\left(x^{\prime}\right) d x^{\prime}\right) \\
& \qquad\left(\begin{array}{c}
\ln a_{1}(k) \\
\ln a_{2}(k)
\end{array}\right)=\left(\begin{array}{c}
d_{1}(k) \\
d_{2}(k)
\end{array}\right)+\mathbf{K} *\left(\begin{array}{c}
\ln A_{1}(k) \\
\ln A_{2}(k)
\end{array}\right)
\end{aligned}
$$

with $d_{j}(k)=-\beta\left(k^{2}+\mu+(-1)^{j} H\right)$ and kernel matrices

$$
\mathbf{K}_{B B}=\left(\begin{array}{cc}
K_{0} & K_{2} \\
K_{1} & K_{0}
\end{array}\right), \quad \mathbf{K}_{F F}=\left(\begin{array}{cc}
0 & K_{2} \\
K_{1} & 0
\end{array}\right),
$$

for the $2 \mathrm{CBG}$ and $2 \mathrm{CFG}$ and

$$
\mathbf{K}_{B F}=\left(\begin{array}{cc}
K_{0} & K_{2} \\
K_{1} & 0
\end{array}\right)
$$

for the Bose-Fermi mixture. It is therefore tempting to conjecture that the thermodynamics of a threecomponent system with contact interactions can be described by three auxiliary functions $a_{i}(k), i=$ $1,2,3, A_{i}(k)=1+a_{i}(k)$, with grand canonical potential

$\phi\left(\left\{\mu_{i}\right\}_{i=1}^{3}, \beta\right)=-\frac{1}{2 \pi \beta} \int_{\mathbb{R}} \ln A_{1}(k)+\ln A_{2}(k)+\ln A_{2}(k) d k$ and $a_{i}(k)$ satisfying

$$
\left(\begin{array}{l}
\ln a_{1}(k) \\
\ln a_{2}(k) \\
\ln a_{3}(k)
\end{array}\right)=\left(\begin{array}{c}
d_{1}(k) \\
d_{2}(k) \\
d_{3}(k)
\end{array}\right)+\mathbf{K} *\left(\begin{array}{c}
\ln A_{1}(k) \\
\ln A_{2}(k) \\
\ln A_{3}(k)
\end{array}\right)
$$

with $d_{j}(k)=-\beta\left(k^{2}+\mu_{j}\right)$. In the case of a threecomponent bosonic and fermionic system we conjecture that the kernels are

$\mathbf{K}_{B B B}=\left(\begin{array}{ccc}K_{0} & K_{2} & K_{2} \\ K_{1} & K_{0} & K_{2} \\ K_{1} & K_{1} & K_{0}\end{array}\right), \quad \mathbf{K}_{F F F}=\left(\begin{array}{ccc}0 & K_{2} & K_{2} \\ K_{1} & 0 & K_{2} \\ K_{1} & K_{1} & 0\end{array}\right)$

and in the case of the Bose-Bose-Fermi and Bose-FermiFermi mixtures the kernels are

$\mathbf{K}_{B B F}=\left(\begin{array}{ccc}K_{0} & K_{2} & K_{2} \\ K_{1} & K_{0} & K_{2} \\ K_{1} & K_{1} & 0\end{array}\right), \quad \mathbf{K}_{B F F}=\left(\begin{array}{ccc}K_{0} & K_{2} & K_{2} \\ K_{1} & 0 & K_{2} \\ K_{1} & K_{1} & 0\end{array}\right)$

These conjectured thermodynamic descriptions present the correct limits when $c \rightarrow 0$ and when one of the components is suppressed, however, a definitive proof of their validity requires the numerical checking with the TBA predictions. This will be addressed in a future publication.

\section{CONTACT}

The momentum distribution of $1 \mathrm{D}$ models with contact interactions present a universal $n(k) \sim \mathcal{C} / k^{4}$ decay [81, 90, 91. The universal coefficient, $\mathcal{C}$, which governs the asymptotic behavior is called the contact and appears in a series of identities involving macroscopic properties of the system which are called Tan relations [78, 91]. The $1 / k^{4}$ decay and the Tan relations are valid also for nonintegrable systems in the presence of a trapping potential, at zero or finite temperature and for few- or many-body systems. For the BFM the bosonic and fermionic contacts are given by [51, 91.

$$
\begin{aligned}
& \mathcal{C}_{B}=c^{2}\left(\left\langle\Psi_{B}^{\dagger} \Psi_{B}^{\dagger} \Psi_{B} \Psi_{B}\right\rangle+\left\langle\Psi_{B}^{\dagger} \Psi_{F}^{\dagger} \Psi_{F} \Psi_{B}\right\rangle\right), \\
& \mathcal{C}_{F}=c^{2}\left\langle\Psi_{B}^{\dagger} \Psi_{F}^{\dagger} \Psi_{F} \Psi_{B}\right\rangle .
\end{aligned}
$$

Even though the individual contacts are hard to compute the total contact can be derived from the thermodynamics of the system using the Hellmann-Feynman theorem 91

$$
\mathcal{C}=\mathcal{C}_{B}+\mathcal{C}_{F}=c^{2}\left(\frac{\partial \phi}{\partial c}\right)_{\mu, H, T}
$$

\section{A. Contact at zero temperature}

At zero temperature the thermodynamics of the system is described by a system of Fredholm integral equations which can be derived from the BAEs (3) 24, 25]

$$
\begin{aligned}
& \rho_{c}(k)=\frac{1}{2 \pi}+\int_{-\lambda_{0}}^{\lambda_{0}} b_{1}(k-\lambda) \rho_{s}(\lambda) d \lambda, \\
& \rho_{s}(\lambda)=\int_{-k_{0}}^{k_{0}} b_{1}(\lambda-k) \rho_{c}(k) d k .
\end{aligned}
$$

Here $k_{0}$ and $\lambda_{0}$ are two parameters which fix the total density $n=M / L_{B F}$ and the boson fraction $\alpha=$ $M_{B} / L_{B F}$ via $n=\int_{-k_{0}}^{k_{0}} \rho_{c}(k) d k$, and $\alpha=\int_{-\lambda_{0}}^{\lambda_{0}} \rho_{s}(\lambda) d \lambda$. The energy density of the system is $\mathcal{E}=\int_{-k_{0}}^{k_{0}} k^{2} \rho_{c}(k) d k$. It is useful to introduce the dimensionless coupling strength $\gamma=c / n$. The system is in the Tonks-Girardeau regime when $\gamma \gg 1$ and weakly interacting when $\gamma \ll 1$.

Once we have computed the energy density the total contact can be derived from Eq. (14) which at zero temperature takes the form

$$
\mathcal{C}=n \gamma^{2}\left(\frac{\partial \mathcal{E}}{\partial \gamma}\right)_{n, \alpha} .
$$

In general it is relatively easy to derive approximate expressions for the energy in the strong coupling limit [24, 25, 51 .

$$
\begin{aligned}
\mathcal{E}_{S}(\gamma, \alpha) \underset{\gamma \gg 1}{\sim} \frac{n^{3} \pi^{2}}{3}[1- & \frac{4}{\gamma}\left(\alpha+\frac{\sin \pi \alpha}{\pi}\right) \\
& \left.+\frac{12}{\gamma^{2}}\left(\alpha+\frac{\sin \pi \alpha}{\pi}\right)^{2}\right],
\end{aligned}
$$

however, in the weakly interacting limit serious difficulties are encountered due to the fact that the $b_{1}(k)$ kernel 

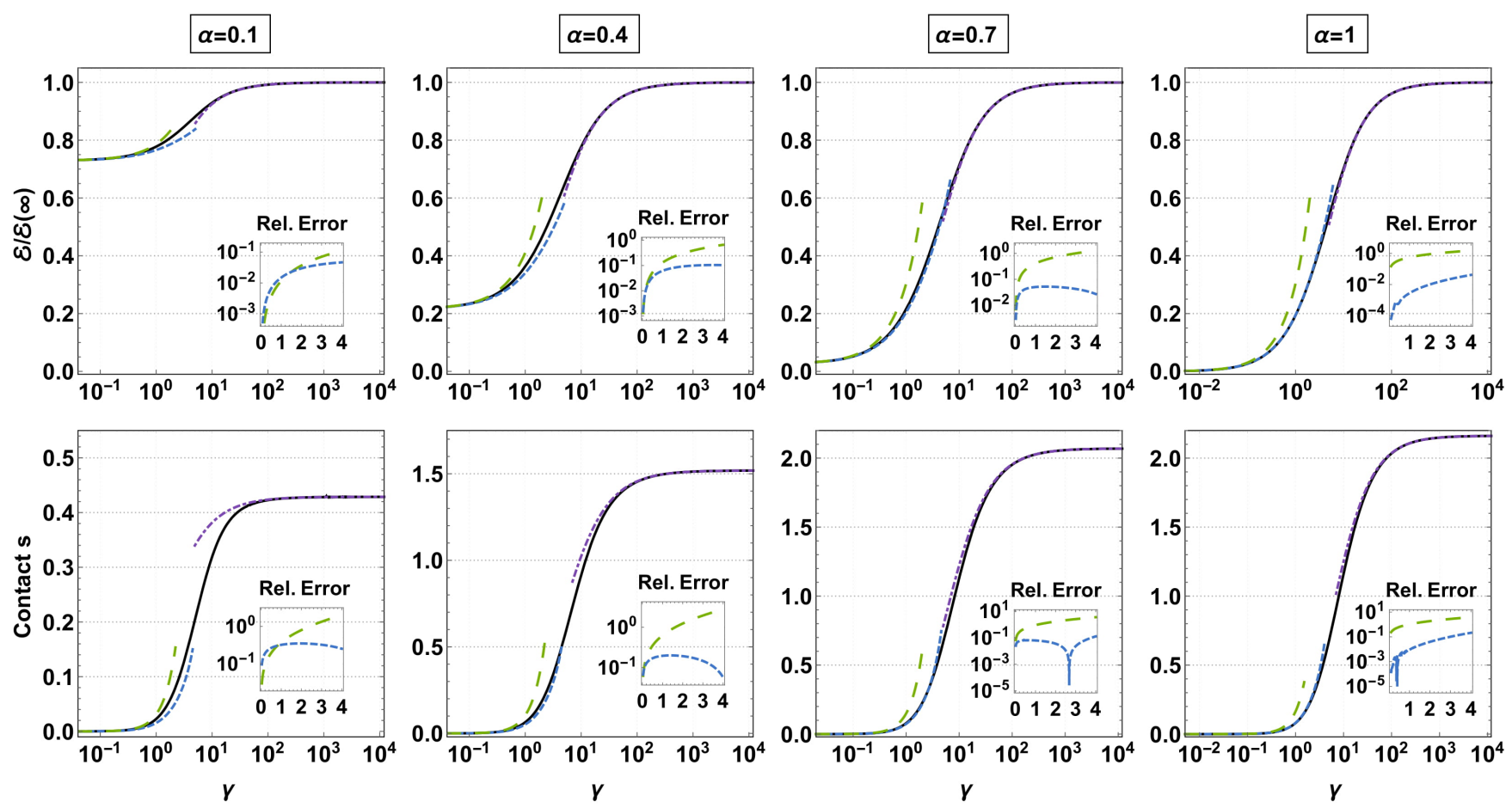

FIG. 2. Upper panels. Energy density normalized by $\mathcal{E}(\infty)=n^{3} \pi^{2} / 3$ (black continuous line) as a function of the dimensionless coupling strength $\gamma$ for several values of the boson fraction. Also plotted are the strong and weak coupling approximations given by Eq. 17) (violet dash dotted line), Eq. 118) (long dashed green line) and Eq. (19) (short dashed blue line). The insets contain the relative errors $\left|\mathcal{E}-\mathcal{E}_{W, W I}\right| / \operatorname{Max}\left[\mathcal{E}, \mathcal{E}_{W, W I}\right]$ of the weak coupling expansions which shows that $(19)$ is an improved approximation. The density is fixed $n=1 / 2$. Lower panels. Normalized total contact $s=\mathcal{C} /(\pi n)^{4}$ as a function of the coupling strength derived from the expressions for the energy and approximations using Eq. (16). The insets contain the relative errors of the contacts derived from the two weak coupling expansions.
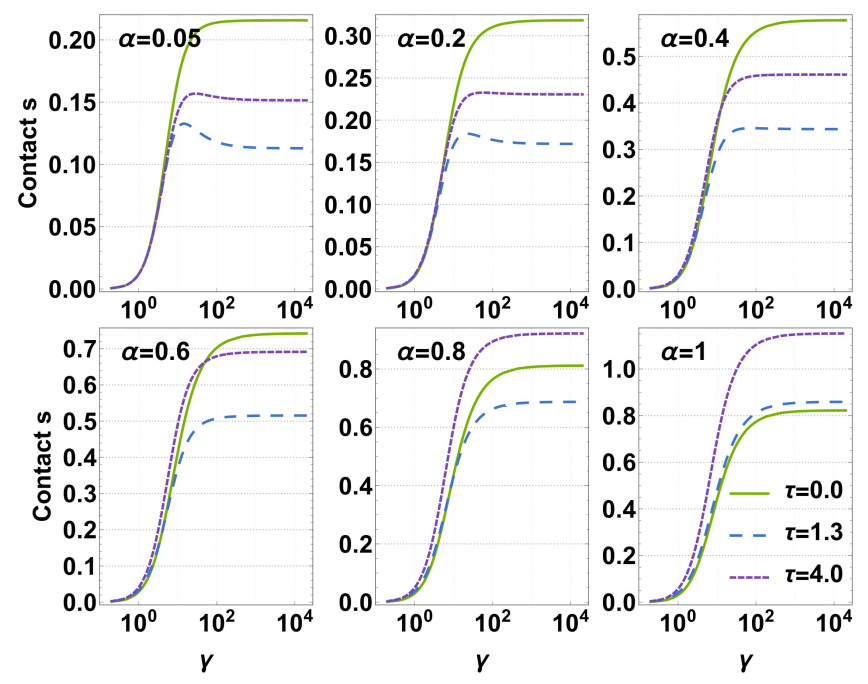

FIG. 3. Dependence of the dimensionless contact on the coupling strength $\gamma$ for several values of the reduced temperature $\left(\tau=T / n^{2}, n=1 / 2\right)$ and different boson fractions. Compared with the ground state, the contact develops a local maximum for small values of $\alpha$, which is more pronounced at low but finite temperatures. becomes a delta function. In this limit only the first term of the asymptotic expansion was obtained [5]

$$
\mathcal{E}_{W}(\gamma, \alpha) \underset{\gamma \ll 1}{\sim} n^{3}\left[\frac{\pi^{2}}{3}(1-\alpha)^{3}+2 \gamma \alpha-\gamma \alpha^{2}\right] .
$$

One way in which we can improve this approximate expression is to replace the $\gamma$ terms which are multiplied with powers of the boson fraction with the weak coupling expansion of the Lieb-Liniger model $93 \quad 97$. $\mathcal{E}_{L L}(\gamma) \underset{\gamma \ll 1}{\sim} \gamma-\frac{4}{3 \pi} \gamma^{3 / 2}+\left(\frac{1}{6}-\frac{1}{\pi^{2}}\right) \gamma^{2}$, obtaining

$\mathcal{E}_{W I}(\gamma, \alpha) \underset{\gamma \ll 1}{\sim} n^{3}\left[\frac{\pi^{2}}{3}(1-\alpha)^{3}+2 \mathcal{E}_{L L}(\gamma) \alpha-\mathcal{E}_{L L}(\gamma) \alpha^{2}\right]$.

This expression reduces to the free fermionic result for $\alpha=0$ and reproduces the Lieb-Liniger expansion when the system is purely bosonic $(\alpha=1)$. In the upper panels of Fig. 2 we present results for the normalized energy density computed using (15) together with the asymptotic expansions at strong and weak coupling. The insets show that 19 represents a significant improvement over (18) and for $\alpha>0.5$ the asymptotic expansions are valid for almost all values of the coupling strengths. The dimensionless contact $s=\mathcal{C} /(\pi n)^{4}$ calculated using (16) 

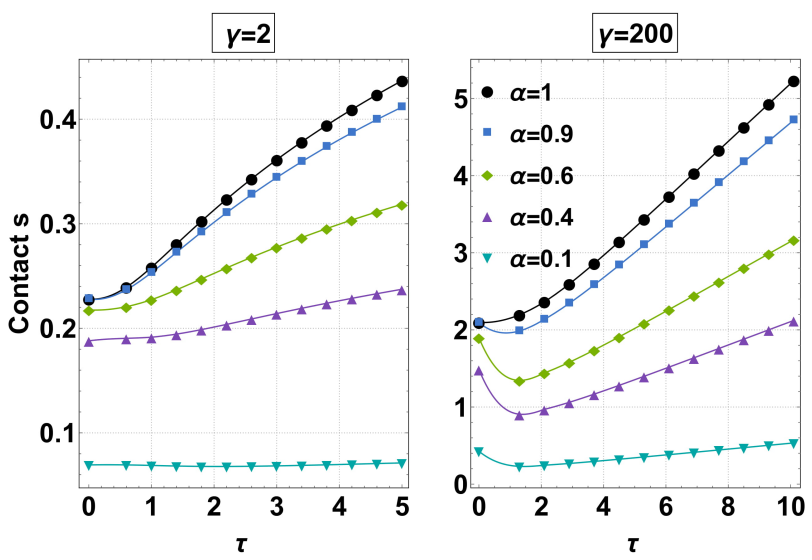

FIG. 4. Dependence of the dimensionless contact on the reduced temperature for $\gamma=2$ and $\gamma=200$. At strong coupling the contact presents a pronounced minimum for all values of the boson fraction except $\alpha=1$.

is shown in the lower panels of Fig. 2, At zero temperature the contact is a monotonically increasing function of both coupling constant and bosonic fraction.

\section{B. Contact at finite temperature}

At finite temperature we use (6), (7) and (14) for the determination of the contact. The dependence of the contact on the coupling strength for $\tau=0,1.3,4$ with $\tau=T / n^{2}$ and different boson fractions is shown in Fig. 3 . We distinguish two notable features. First, for small values of the boson fraction, $\alpha=0.05$ and $\alpha=0.2$, the contact at finite temperatures develops a local maximum which is more pronounced at low temperatures. Second, with the exception of the system close to the purely bosonic case, $\alpha=1$, for large values of the coupling strength the contact at zero temperature is larger than the one at finite temperature. This is rather counterintuitive if we remember that the contact governs the long tail of the momentum distribution. Therefore, a smaller contact at higher temperature means that as we increase $T$ the number of particles with large momenta decreases compared with the ground state. This phenomenon can be seen more clearly in Fig. 4 where we present the dependence of the contact on the reduced temperature for moderate and strong coupling. For $\gamma=2$ the contact is a monotonically increasing function of the temperature for all values of the boson fraction, however, at strong coupling the contact develops a pronounced minimum the only exception being the case of $\alpha=1$. This momentum reconstruction at low temperatures is a feature of $1 \mathrm{D}$ multi-component systems being present also in the case of the two-component Fermi [77] and Bose [92] gas and serves as a signature of the transition from the Tomonaga-Luttinger liquid phase to the spin-incoherent regime. In 1D two-component systems there are two relevant temperature scales [98]: the Fermi temperature $T_{F}=\pi^{2} n^{2}$ which characterizes the charge degrees of freedom and $T_{0}=E_{F} / \gamma$ which estimates the bandwidth of the spin excitations (in our case a "spin excitation" is represented by the removal of a fermion and the addition of a boson in the system). In the strong coupling limit we have $0 \ll T_{0} \equiv E_{F} / \gamma \ll E_{F}$ and for $T \in\left(T_{0}, T_{F}\right)$ the charge degrees of freedom are effectively frozen while the spin degrees of freedom are highly excited. This regime is called spin-incoherent 99 103. and its properties are significantly different from the more well known Tomonaga-Luttinger liquid phase. In the BFM the minima of the contact at the transition point out that the momentum distribution becomes narrower but is also easy to see that this is also accompanied by significant changes at low momenta. In the TLL regime the Bose-Bose field correlator presents algebraic decay with $\left\langle\Psi_{B}^{\dagger}(x) \Psi_{B}(0)\right\rangle \sim 1 /|x|^{-1 /\left(2 K_{b}\right)}$ with $K_{b}=1 /\left[(\alpha-1)^{2}-1\right]$ derived by Frahm and Palacios [1] and numerically confirmed in 25]. Therefore, the bosonic momentum distribution will have a singularity at $k=0$ of the type $n_{B}(k) \sim 1 /|k|^{-1+1 /\left(2 K_{b}\right)}$. However, in the spin-incoherent regime the correlators are exponentially decaying which means that the momentum distribution at zero becomes finite. This shows that there is a significant momentum reconstruction both at low and large momenta at the transition between the TLL and spinincoherent regime.

\section{BOUNDARIES OF THE QUANTUM CRITICAL REGIONS}

In the vicinities of the quantum critical points (QCP) the thermodynamics of the system is universal and is determined by the universality class of the quantum phase transition. If we keep the magnetic field fixed and consider the chemical potential as driving parameter, in the quantum critical region the pressure can be written as 104

$$
p(\mu, H, T) \sim p_{r}(\mu, H)+T^{\frac{d}{z}+1} \mathcal{P}_{H}\left(\frac{\mu-\mu_{c}(H)}{T^{\frac{1}{\nu z}}}\right),
$$

with $p_{r}$ the regular part of the pressure, $d$ the dimension, $\mathcal{P}_{H}$ a universal function and $\mu_{c}(H)$ the quantum critical point. The universality class of the transition is determined by the correlation length exponent $\nu$ and the dynamical critical exponent $z$. All the other thermodynamic quantities can be derived from (20). For example, the density and compressibility which are defined by $n=\partial p / \partial \mu$ and $\kappa=\partial^{2} \phi / \partial \mu^{2}$ are

$$
\begin{aligned}
n(\mu, H, T) & \sim \frac{\partial p_{r}}{\partial \mu}(\mu, H)+T^{\frac{d}{z}+1-\frac{1}{\nu z}} \mathcal{P}_{H}^{\prime}\left(\frac{\mu-\mu_{c}(H)}{T^{\frac{1}{\nu z}}}\right), \\
\kappa(\mu, H, T) & \sim \frac{\partial^{2} p_{r}}{\partial \mu^{2}}(\mu, H)+T^{\frac{d}{z}+1-\frac{2}{\nu z}} \mathcal{P}_{H}^{\prime \prime}\left(\frac{\mu-\mu_{c}(H)}{T^{\frac{1}{\nu z}}}\right) .
\end{aligned}
$$



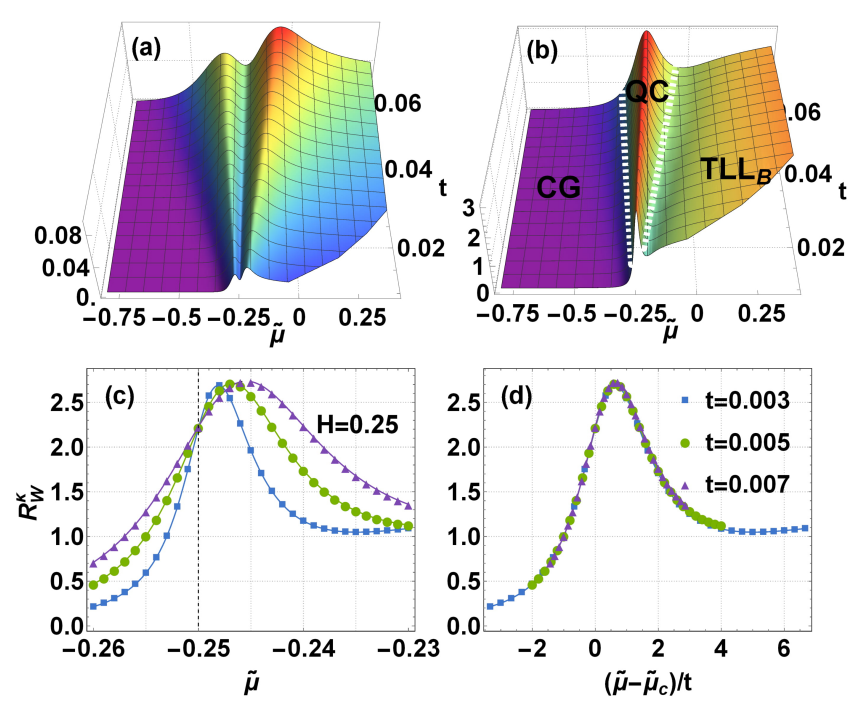

FIG. 5. (a) 3D plot of the grand canonical specific heat for $c=1$ and $H=0.25$ as a function of the chemical potential and temperature $\left(\tilde{\mu}=\mu / c^{2}, t=T / c^{2}\right)$. The lines of local maxima fanning out from the QCP, $\tilde{\mu}_{c}=-H / c^{2}$, are the boundaries of the $\mathrm{QC}$ region. (b) $3 \mathrm{D}$ plot of the Wilson ratio. The white dashed lines are the boundaries of the critical region. CG represents the vacuum (classical gas) phase and $T L L_{B}$ is the Tomonaga-Luttinger liquid phase of single component bosons. (c) Plot of the Wilson ratio as a function of the chemical potential for three values of temperature. All the curves intersect at the QCP (dashed vertical line). The critical exponents are $z=2$ and $\nu=1 / 2$. (d) When plotted as a function of $\left(\tilde{\mu}-\tilde{\mu}_{c}(H)\right) / t$ all the curves collapse to the universal function $\mathcal{Q}_{H}$ (see Eq. 22 .

We can determine the universality class of the transition by choosing certain values for $z$ and $\nu$ and plotting the scaled pressure $\left(p-p_{r}\right) T^{-\frac{d}{z}-1}$ for several values of temperature 104. If we have chosen correctly the exponents all the curves will intersect at the value of the QCP $\mu_{c}(H)$. If we plot the scaled pressures as a function of $\left(\mu-\mu_{c}(H)\right) / T^{\frac{1}{\nu z}}$ all the curves should collapse to the universal curve $\mathcal{P}_{H}$.

A problem of considerable importance, both theoretically and experimentally, is the determination of the boundaries of the critical regions. The properties of the system in the CR are fundamentally different from the ones of other low-temperature phases and are characterized by the strong coupling of quantum and thermal fluctuations. In 92, 105 107] it was argued that the grand canonical specific heat, $c_{V}=-T \partial^{2} \phi / \partial T^{2}$ can be used to determine the boundaries of the $\mathrm{QC}$ regions with great precision. This is due to the fact that the grand canonical specific heat is related to both the energy and number of particles fluctuations via $k_{B} T^{2} c_{v}=\left\langle\delta(E-\mu N)^{2}\right\rangle$ which means that the $\mathrm{QC}$ boundaries can be identified with the local maxima of this quantity. Another important quantity which can be used to identify the low temperature phases is the compressibility Wilson ratio [92, 108, 109]
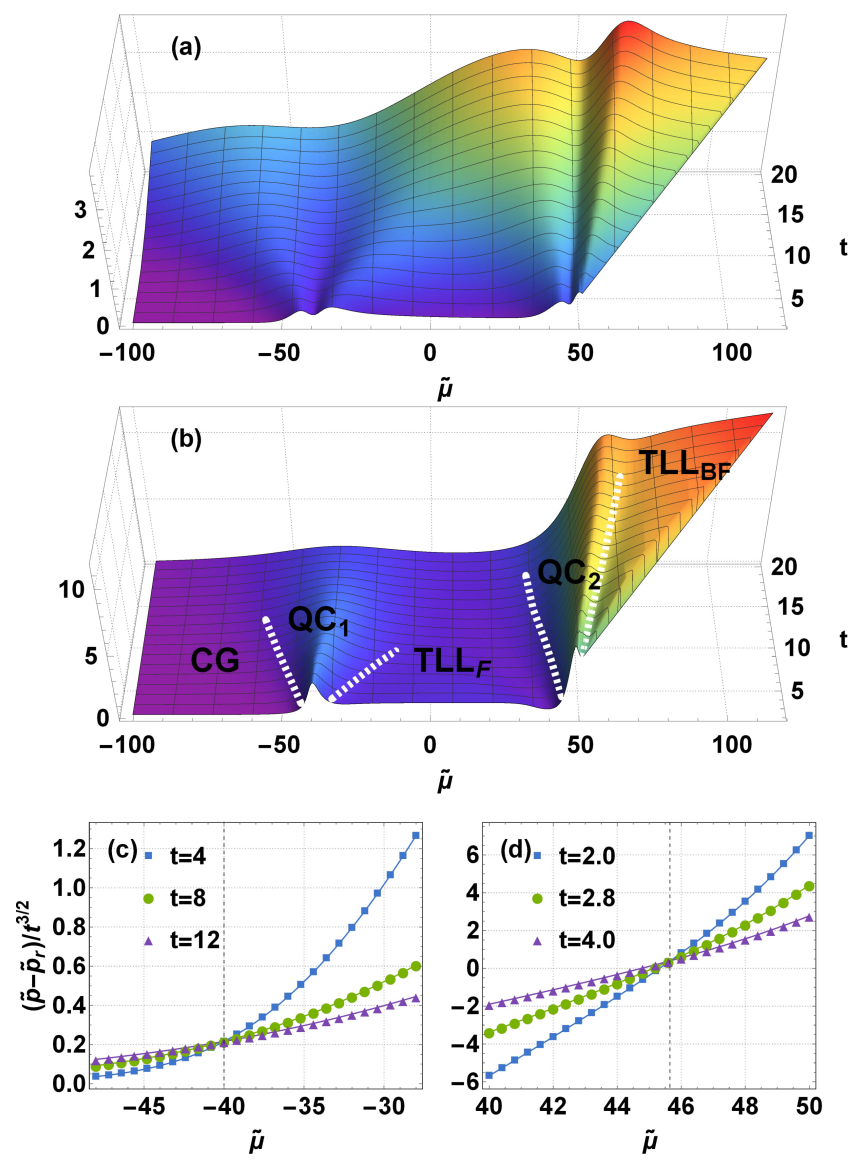

FIG. 6. (a) 3D plot of the grand canonical specific heat for $c=0.05$ and $H=-0.1\left(\tilde{\mu}=\mu / c^{2}, t=T / c^{2}\right)$. In this case we have two sets of lines of local maxima which determine the boundaries of the $\mathrm{QC}$ regions emerging from the quantum critical points situated at $\tilde{\mu}_{c}^{(1)}=-|H| / c^{2}$, and $\tilde{\mu}_{c}^{(2)} \sim 0.114 / c^{2}$. (b) $3 \mathrm{D}$ plot of the Wilson ratio. The white dashed lines represent the boundaries of the critical regions $Q C_{1}$ and $Q C_{2}$. CG, $T L L_{F}$ and $T L L_{B F}$ stand for the classical gas phase, TLL phase of single component fermions and TLL phase of bosons and fermions, respectively. (c) Scaled pressure $\left(\tilde{p}=p / c^{3}\right)$ as a function of the chemical potential for three temperatures in the vicinity of the first QCP. For $z=2$ and $\nu=1 / 2$ all the curves intersect at $\tilde{\mu}_{c}^{(1)}=-|H| / c^{2}$. (d) Scaled pressure in the vicinity of the second QCP. For $z=2$ and $\nu=1 / 2$ all the curves intersect at $\tilde{\mu}_{c}^{(2)} \sim 0.114 / c^{2}$.

defined by

$$
R_{W}^{\kappa}=\frac{\pi^{2} k_{B}^{2}}{3} T \frac{\kappa}{c_{V}}
$$

with $\kappa$ the compressibility. Because $k_{B} T \kappa=\left\langle\delta N^{2}\right\rangle$ the Wilson ratio will be almost constant in the lowtemperature phases and will present anomalous enhancement in the QC regions and will scale like [108]

$$
R_{W}^{\kappa} \sim \mathcal{Q}_{H}\left(\frac{\mu-\mu_{c}(H)}{T^{\frac{1}{\nu z}}}\right)+w_{0} T^{1 / 2} \mathcal{F}_{H}\left(\frac{\mu-\mu_{c}(H)}{T^{\frac{1}{\nu z}}}\right) .
$$


In the previous equation $\mathcal{Q}_{H}$ and $\mathcal{F}_{H}$ are two universal functions, $w_{0}$ is a constant and the second term in the right hand side appears only if $p_{r}$ is nonzero.

The quantum critical points and the phase diagram at zero temperature were determined in 31 . 11 The number of QCPs depends on the sign of the magnetic field. For $H>0$ we have only a QPT from the vacuum to a single component TLL with critical point $\mu_{c}=-H$. In Fig. 5 (a) we present results for the dependence of the grand canonical specific heat on temperature and chemical potential for $H=0.25$ and coupling strength $c=1$. The specific heat presents two lines of local maxima fanning out from the QCP which separate the vacuum (classical gas) and the TLL phase from the QC region. The Wilson ratio, depicted in Fig. 5 (b), is zero in the classical gas phase presents a local maximum in the $\mathrm{QC}$ region and is slowly increasing in the TLL phase. In this case $p_{r} \sim 0$ and $R_{W}^{\kappa}$ obeys the scaling relation 20 with only the first term on the right hand side. The scaling and collapse of the curves to the universal function $\mathcal{Q}_{H}$ is realized for $z=2$ and $\nu=1 / 2$ and is presented in Fig. 5 (c) and Fig. 5 (d). The value of the critical exponents would seem to point out that this QPT is in the universality class of free fermions. However it was argued in 92 that in fact this QPT belongs to the universality class of spindegenerate impenetrable particle gas with the universal thermodynamics described by Takahashi's formula 66 $(x=(\mu+|H|) / T, y=H / T)$

$$
p=\frac{T^{3 / 2}}{2 \pi} \int_{-\infty}^{+\infty} \ln \left[1+\left(1+e^{-2|y|}\right) e^{-k^{2}+x}\right] d k,
$$

in contrast with the free fermionic case for which $\left(x^{\prime}=\right.$ $\mu / T)$,

$p_{F F}=\frac{T^{3 / 2}}{2 \pi} \int \ln \left[\left(1+e^{-k^{2}+x^{\prime}+y}\right)\left(1+e^{-k^{2}+x^{\prime}-y}\right)\right] d k$.

In the case of fixed negative magnetic field there are two QPTs. The first QCP is $\mu_{c}^{(1)}=-|H|$ where the system has a phase transition from the vacuum to a TLL phase of single component fermions. The value of the second QCP is determined by $\left(\tilde{\mu}_{c}^{(2)}=\left(\mu_{c}^{(2)}-H\right) / c^{2}\right)$ 31]

$-\frac{2 H}{c^{2}}=\frac{1}{2 \pi}\left[\left(1+4 \tilde{\mu}_{c}^{(2)}\right) \arctan \left(4 \tilde{\mu}_{c}^{(2)}\right)^{1 / 2}-\left(4 \tilde{\mu}_{c}^{(2)}\right)^{1 / 2}\right]$,

where we have a QPT between the single component fermionic TLL to a two-component TLL composed of fermions and bosons. The boundaries of the two QC regions for $c=0.05$ and $H=-0.1$ identified with the

\footnotetext{
${ }^{1}$ It should be noted that the definitions of the chemical potential and effective magnetic field employed by us are different from the ones used in 31] which will be denoted by the $Y G Z G$ subscript. We have $\mu=\mu_{Y G Z C}$ and $H=-H_{Y G Z C} / 2$.
}

maxima of the specific heat are shown in Fig. 6 (a) and Fig. 6 (b). In the case of single component systems with QPT belonging to the free fermionic universality class Maeda et al. 110 derived a universal relation which determines the boundary between the QC and TLL regions. For $H \gg T$ this relation is also valid for the first QPT of the BFM due to the fact that in this regime Takahashi's formula 23 is equivalent to the pressure of single component free fermions. We stress that the identification of CR boundaries using the maxima of the specific heat has the advantage of identifying both boundaries in addition to being valid also for multi-component systems.

The Wilson ratio presents anomalous enhancement in both critical regions. For single component systems TLL theory predicts that $R_{W}^{\kappa}=K$ [106, 111] with $K$ the TLL parameter relation which was "experimentally verified" in the Lieb-Liniger model 106. This identity is also valid for the Bose-Fermi mixture in the TLL regime of the first QPT for $H \gg T$.

The critical exponents of both QPTs are $z=2, \nu=$ $1 / 2$ as shown in Fig. 6(c) and Fig. 6. (d) where the curves for the scaled pressure at different temperatures intersect at $\mu_{c}^{(1)}=-|H|$ for the first QPT and at $\mu_{c}^{(2)}=$ $0.114118 \cdots$ for the second QPT. While the first transition is in the spin-degenerate universality class characterized by Eq. (23) it is surprising that the second QPT has the same critical exponents as the free fermionic universality class 112 . We point out that the true universal thermodynamics 23 in the vicinity of the critical point $(\mu, H)=(0,0)$ is different from the free spinor fermion thermodynamics 24. In the case of the first transition, (23) and (24) agree, for $H \gg T$. For the second critical line it is possible that the universal thermodynamics is described by a scaling function different from 23) or (24).

Lastly, we like to point out certain similarities of the zero temperature phase diagram of the Bose-Fermi system with those of the pure Bose-Bose and Fermi-Fermi systems with otherwise same mass and interaction parameters. For $H \geq 0$ the $\mathrm{BF}$ phase diagram is identical to that of the BB system with vacuum phase for $\mu<\mu_{c}$ and completely polarized bosonic phase for $\mu>\mu_{c}$. Viewed from $H>0$, the line $\mu>0, H=0$ is a transition line into a mixed phase. The location of this line is given by the single particle properties of the new admixed particle, the line does not depend on its statistics.

For $H<0$ the $\mathrm{BF}$ phase diagram is identical to that of the FF system with vacuum phase for $\mu<\mu_{c}^{(1)}$, completely polarized fermionic phase for $\mu_{c}^{(1)}<\mu<\mu_{c}^{(2)}$, and mixed fermionic-bosonic phase for $\mu_{c}^{(2)}<\mu$. The critical line $\mu_{c}^{(2)}=\mu_{c}^{(2)}(H)$ satisfies 25 for the $\mathrm{BF}$ and the $\mathrm{FF}$ case as can be derived from the low temperature limit of the TBA equations for the BF case [31] as well as for the FF case [113. When approaching this line from the polarized phase, its location is again given by the single particle properties of the new admixed particle, the line does not depend on its nature. 


\section{THE BOSE-FERMI MIXTURE AS THE CONTINUUM LIMIT OF THE PERK-SCHULTZ SPIN CHAIN}

The derivation of the BFM's thermodynamic description, (6) and (7), consists of three steps. First, we show that the Perk-Schultz spin chain 114 119 is a lattice embedding of our continuum model. The thermodynamics of the spin-chain is then investigated with the quantum transfer matrix technique [69 74] which relates the free energy of the model to the largest eigenvalue of the QTM and involves only a finite number of NLIEs. Finally, the result for the BFM is obtained by taking the continuum limit in the lattice result. This method was first employed in the case of the Lieb-Liniger model [120] and then used to derive efficient, that is involving only a finite number of NLIEs, thermodynamic descriptions for the 2CBG [75, 76] and 2CFG [77]. Because the ratios of the largest to the next-largest eigenvalues of the QTM give the correlation lengths of various Green's function the same algorithm can be used to investigate the asymptotic behavior of correlators in integrable continuum models [121, 122.

As in the case of the $2 \mathrm{CBG}$ and $2 \mathrm{CFG}$ the lattice embedding of the Bose-Fermi mixture is the critical $q=3$ Perk-Schultz spin-chain [114 119], the only difference being the grading, which in this case is $(-+-$ ) (see also [76, 77]). Here, by a lattice embedding we understand a lattice model whose spectrum and BAEs transform under a suitable scaling limit in the spectrum and BAEs of the continuum model. The Hamiltonian for an arbitrary grading $\left(\varepsilon_{1}, \varepsilon_{2}, \varepsilon_{3}\right),\left(\varepsilon_{i} \in\{ \pm 1\}\right)$ is

$$
\mathcal{H}_{\mathcal{P S}}=J \varepsilon_{1} \sum_{j=1}^{L}\left(\cos \gamma \sum_{a=1}^{3} \varepsilon_{a} e_{a a}^{(j)} e_{a a}^{(j+1)}+\sum_{\substack{a, b=1 \\ a \neq b}}^{3} e_{a b}^{(j)} e_{b a}^{(j+1)}+i \sin \gamma \sum_{\substack{a, b=1 \\ a \neq b}}^{3} \operatorname{sign}(a-b) e_{a a}^{(j)} e_{b b}^{(j+1)}\right)-\sum_{j=1}^{L} \sum_{a=1}^{3} h_{a} e_{a a}^{(j)}
$$

with $L$ the number of lattice sites, $J>0$ the coupling strength and $h_{1}, h_{2}, h_{3}$ chemical potentials. Also, in (26) $\gamma \in[0, \pi]$ is the anisotropy (not to be confused with the dimensionless coupling constant of the continuum model) and $e_{a b}^{(j)}=\mathbb{I}_{3}^{\otimes j-1} \otimes e_{a b} \otimes \mathbb{I}_{3}^{\otimes L-j}$, with $e_{a b}$ and $\mathbb{I}_{3}$ the canonical basis and the unit matrix in the space of 3-by-3 matrices. For the $(-+-)$ grading the energy spectrum is

$$
E_{P S}=\sum_{j=1}^{M} e_{0}\left(v_{j}^{(1)}\right)+M_{1}\left(h_{2}-h_{3}\right)+E_{0}, \quad E_{0}=J L \cos \gamma-h_{1} L, \quad e_{0}(v)=\frac{J \sin ^{2} \gamma}{\sin (v-\gamma) \sin v},
$$

with $\left\{v_{s}^{(1)}\right\}_{s=1}^{M}$ and $\left\{v_{l}^{(2)}\right\}_{l=1}^{M_{1}}$ satisfying the BAEs

$$
\begin{array}{ll}
\left((-1) \frac{\sin \left(v_{s}^{(1)}-\gamma\right)}{\sin v_{s}^{(1)}}\right)^{L}=(-1)^{M-1} \prod_{p=1}^{M_{1}} \frac{\sin \left(v_{s}^{(1)}-v_{p}^{(2)}-\gamma\right)}{\sin \left(v_{s}^{(1)}-v_{p}^{(2)}\right)}, & s=1 \cdots, M, \\
\prod_{j=1}^{M} \frac{\sin \left(v_{l}^{(2)}-v_{j}^{(1)}+\gamma\right)}{\sin \left(v_{l}^{(2)}-v_{j}^{(1)}\right)}=(-1)^{M_{1}-1}, & l=1, \cdots, M_{1} .
\end{array}
$$

First, we will show how we can obtain $(3)$ from 28. We consider $v_{s}^{(1)} \rightarrow i \delta k_{s}^{(1)} / \epsilon+\gamma / 2$ and $v_{s}^{(2)} \rightarrow i \delta k_{s}^{(2)} / \epsilon+\pi / 2$ with $\epsilon \rightarrow 0$ and lattice constant $\delta \rightarrow O\left(\epsilon^{2}\right)$. Under this transformation 28 become

$$
\begin{array}{cc}
\left((-1) \frac{\sinh \left(\delta k_{s}^{(1)} / \epsilon-i \gamma / 2\right)}{\sinh \left(\delta k_{s}^{(1)} / \epsilon+i \gamma / 2\right)}\right)^{L}=(-1)^{M-1} \prod_{p=1}^{M_{1}} \frac{\cosh \left(\delta k_{s}^{(1)} / \epsilon-\delta k_{p}^{(2)} / \epsilon-i \gamma / 2\right)}{\cosh \left(\delta k_{s}^{(1)} / \epsilon-\delta k_{p}^{(2)} / \epsilon+i \gamma / 2\right)}, & s=1, \cdots, M, \\
\prod_{j=1}^{M} \frac{\cosh \left(\delta k_{l}^{(2)} / \epsilon-\delta k_{j}^{(1)} / \epsilon-i \gamma / 2\right)}{\cosh \left(\delta k_{l}^{(2)} / \epsilon-\delta k_{j}^{(1)} / \epsilon+i \gamma / 2\right)}=(-1)^{M_{1}-1}, & l=1, \cdots, M_{1} .
\end{array}
$$

In the second step we perform $\gamma \rightarrow \pi-\epsilon$ with the result

$$
\begin{array}{rlr}
\left(\frac{\cosh \left(\delta k_{s}^{(1)}+i \epsilon / 2\right)}{\cosh \left(\delta k_{s}^{(1)}-i \epsilon / 2\right)}\right)^{L} & =(-1)^{M+M_{1}-1} \prod_{p=1}^{M_{1}} \frac{\sinh \left(\delta k_{s}^{(1)} / \epsilon-\delta k_{p}^{(2)} / \epsilon+i \epsilon / 2\right)}{\sinh \left(\delta k_{s}^{(1)} / \epsilon-\delta k_{p}^{(2)} / \epsilon-i \epsilon / 2\right)}, & s=1, \cdots, M, \\
\prod_{j=1}^{M} \frac{\sinh \left(\delta k_{l}^{(2)} / \epsilon-\delta k_{j}^{(1)} / \epsilon+i \epsilon / 2\right)}{\sinh \left(\delta k_{l}^{(2)} / \epsilon-\delta k_{j}^{(1)} / \epsilon-i \epsilon / 2\right)} & =(-1)^{M+M_{1}-1}, & l=1, \cdots, M_{1} .
\end{array}
$$

Taking the limit $L \rightarrow \infty$ such that $L \delta=L_{B F}$, intro-

ducing $c=\epsilon^{2} / \delta$ and using

$$
\frac{\cosh \left(\delta k_{s}^{(1)}+i \epsilon / 2\right)}{\cosh \left(\delta k_{s}^{(1)}-i \epsilon / 2\right)} \sim \frac{1+i \delta k_{s}^{(1)} / 2}{1-i \delta k_{s}^{(1)} / 2}
$$


we see that Eqs. (29) transform into the BAEs of the mixture (3) for $M_{1}+M-1$ even and identifying $M_{1}=$ $M_{B}$. Under the same set of transformations we have

$$
\begin{gathered}
E_{P S}-E_{0}=\sum_{j=1}^{M}\left[J \delta^{2}\left(k_{j}^{(1)}\right)^{2}-J \epsilon^{2}-J \epsilon^{4} / 4+h_{1}-h_{2}\right] \\
+\left(h_{2}-h_{3}\right) M_{1}+O\left(\epsilon^{6}\right) .
\end{gathered}
$$

However, we are interested in the thermodynamical behavior and therefore we can also scale the temperature in the models in order to have $\beta\left(E_{P S}-E_{0}\right) \rightarrow \bar{\beta} E_{B F}$ with $E_{B F}$ given by (2). If we consider $J=1, \beta=\bar{\beta} / \delta^{2}$, $h_{1} \rightarrow O\left(\epsilon^{2}\right)$ such that $\left(J \epsilon^{2}-h_{1}\right) / \delta^{2}$ is finite and $h_{2}, h_{3} \rightarrow$ $O\left(\epsilon^{4}\right)$, we obtain $\beta\left(E_{P S}-E_{0}\right) \rightarrow \bar{\beta} E_{B F}$ with $\mu_{F}=$ $\left.J \epsilon^{2}+J \epsilon^{4} / 4-h_{1}+h_{2}\right) / \delta^{2}$ and $\mu_{B}-\mu_{F}=\left(h_{3}-h_{2}\right) / \delta^{2}$. The scaling limit presented in this section is the same as the one used in the $2 \mathrm{CBG}$ and $2 \mathrm{CFG}$ case (see Table I of [76]) and shows that the thermodynamic behavior of the mixture at all temperatures can be derived from the low temperature thermodynamics of the lattice model.

\section{DERIVATION OF THE THERMODYNAMICS FOR THE PERK-SCHULTZ SPIN-CHAIN}

The free energy of the Perk-Schultz spin-chain can be obtained from the largest eigenvalue of the QTM as $f\left(h_{1}, h_{2}, h_{3}, \beta\right)=-\ln \Lambda_{0}(0) / \beta$. For a given Trotter number, denoted by $N$, the largest eigenvalue of the QTM lies in the $(N / 2, N / 2)$ sector (see Appendix A of [76] or [124 [127]) and can be written as

$$
\Lambda_{0}(v)=\lambda_{1}(v)+\lambda_{2}(v)+\lambda_{3}(v),
$$

with

$$
\lambda_{j}(v)=\phi_{-}(v) \phi_{+}(v) \frac{q_{j-1}\left(v-i \tilde{\epsilon}_{j} \gamma\right)}{q_{j-1}(v)} \frac{q_{j}\left(v+i \tilde{\epsilon}_{j} \gamma\right)}{q_{j}(v)} e^{\beta \tilde{h}_{j}},
$$

where $\left(\tilde{\epsilon}_{1}, \tilde{\epsilon}_{2}, \tilde{\epsilon}_{3}\right)=(--+),\left(\tilde{h}_{1}, \tilde{h}_{2}, \tilde{h}_{3}\right)=\left(h_{3}, h_{1}, h_{2}\right)$, and

$$
\phi_{ \pm}(v)=\left(\frac{\sinh (v \pm i u)}{\sin \gamma}\right)^{N / 2}, u=J \sin \gamma \beta / N .
$$

The $q_{j}(v)$ functions are defined as

$$
q_{j}(v)=\left\{\begin{array}{lr}
\phi_{-}(v), & j=0, \\
\prod_{k=1}^{N / 2} \sinh \left(v-v_{k}^{(j)}\right), & j=1,2, \\
\phi_{+}(v), & j=3,
\end{array}\right.
$$

with $\left\{v_{k}^{(1)}\right\}_{k=1}^{N / 2},\left\{v_{k}^{(2)}\right\}_{k=1}^{N / 2}$ parameters which are called Bethe roots and satisfy the quantum transfer matrix BAEs (see below). If we introduce two auxiliary functions

$\mathfrak{a}_{1}=\frac{\lambda_{1}(v)}{\lambda_{2}(v)}=\frac{\phi_{-}(v+i \gamma)}{\phi_{-}(v)} \frac{q_{1}(v-i \gamma)}{q_{1}(v+i \gamma)} \frac{q_{2}(v)}{q_{2}(v-i \gamma)} e^{\beta\left(h_{3}-h_{1}\right)}$,

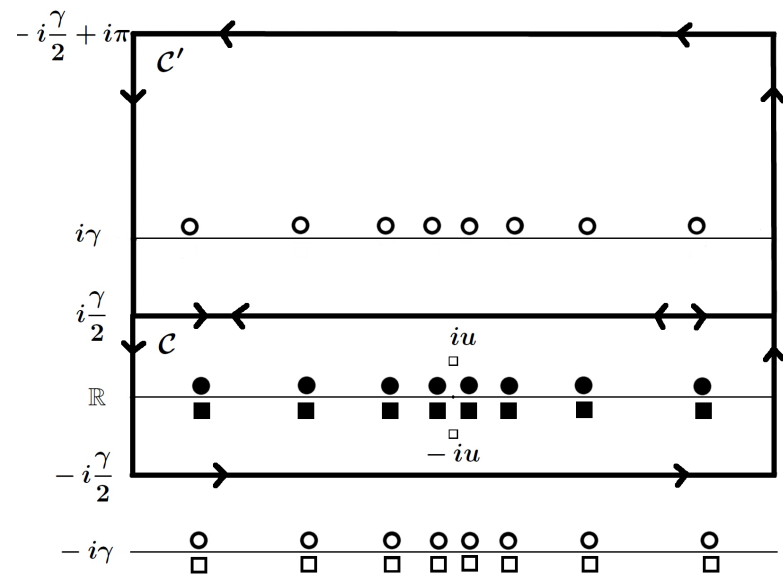

FIG. 7. Distribution of Bethe roots $(\mathbf{\square}, \bullet)$ and holes $(\square, \circ)$ for the largest eigenvalue of the QTM and $\gamma \in(0, \pi / 2)$. The contour $\mathcal{C}$ contains all the Bethe roots and the poles of order $N / 2$ at $\pm i u$. The lower edge of the contour $\mathcal{C}^{\prime}$ coincides with the upper edge of $\mathcal{C}$ but it has opposite orientation.

$\mathfrak{a}_{2}=\frac{\lambda_{3}(v)}{\lambda_{2}(v)}=\frac{\phi_{+}(v+i \gamma)}{\phi_{+}(v)} \frac{q_{1}(v)}{q_{1}(v+i \gamma)} e^{\beta\left(h_{2}-h_{1}\right)}$,

the BAEs of the quantum transfer matrix can be written as $(j=1,2): \mathfrak{a}_{j}\left(v_{k}^{(j)}\right)=-1, k=1, \cdots, N / 2$.

\section{A. Integral equations for the auxiliary functions}

First, we will derive a set of NLIEs for the auxiliary functions (34). Both of the functions are periodic of period $i \pi$. The equation $\mathfrak{a}_{1}(v)=-1$ has $3 N / 2$ solutions, of which $N / 2$ are the so-called Bethe roots, $\left\{v_{j}^{(1)}\right\}_{j=1}^{N / 2}$, and $N$ solutions, which are called holes, and they are denoted by $\left\{{v^{\prime}}_{j}^{(1)}\right\}_{j=1}^{N}$. However, the second equation $\mathfrak{a}_{2}(v)=-1$ has only $N$ solutions, of which $N / 2$ are the Bethe roots, $\left\{v_{j}^{(2)}\right\}_{j=1}^{N}$, and the other $N / 2$ are the second set of holes denoted by $\left\{{v^{\prime}}_{j}^{(2)}\right\}_{j=1}^{N / 2}$. A typical distribution of Bethe roots and holes characterizing the largest eigenvalue of the QTM for $\gamma \in(0, \pi / 2)$ is shown in Fig. 7. For any value of the Trotter number $N$ the strip $|\operatorname{Im} v|<\gamma / 2$ contains all the Bethe roots and the poles of order $N / 2$ at \pm iu. Introducing the rectangular contour $\mathcal{C}$ centered at the origin, which extends to infinity and is depicted in Fig. 7 we can define for $v$ outside of $\mathcal{C}(j=1,2)$

$$
\begin{aligned}
f_{j}(v) & =\frac{1}{2 \pi i} \int_{\mathcal{C}} \frac{d}{d v}[\ln \sinh (v-w)] \ln \left[1+\mathfrak{a}_{j}(w)\right] d w, \\
& =\frac{1}{2 \pi i} \int_{\mathcal{C}} \ln \sinh (v-w) \frac{\mathfrak{a}_{j}^{\prime}(w)}{1+\mathfrak{a}_{j}(w)} d w .
\end{aligned}
$$

The last relation was derived using integration by parts and the fact that the winding number of $\ln \left[1+\mathfrak{a}_{j}(w)\right]$ is 
zero due to the fact that the number of zeroes and order of the poles inside the contour is the same. Then, we can show that (see Sec. 6.3 of [123] or [76, 77])

$$
\begin{aligned}
& f_{1}(v)=\ln q_{1}(v)-\ln \phi_{-}(v)-\frac{N}{2} \ln \sin \gamma \\
& f_{1}(v)=\ln q_{2}(v)-\ln \phi_{+}(v)-\frac{N}{2} \ln \sin \gamma
\end{aligned}
$$

Taking the logarithm of the auxiliary functions (34) and using the previous result 36 we obtain

$$
\begin{aligned}
\ln \mathfrak{a}_{1}(v)= & \beta\left(h_{3}-h_{1}\right)+\ln \left[\frac{\phi_{+}(v)}{\phi_{-}(v)} \frac{\phi_{-}(v-i \gamma)}{\phi_{+}(v-i \gamma)}\right] \\
& +f_{1}(v-i \gamma)-f_{1}(v+i \gamma)+f_{2}(v)-f_{2}(v-i \gamma), \\
\ln \mathfrak{a}_{2}(v)=\beta( & \left.h_{2}-h_{1}\right)+\ln \left[\frac{\phi_{-}(v)}{\phi_{+}(v)} \frac{\phi_{+}(v+i \gamma)}{\phi_{-}(v+i \gamma)}\right] \\
& +f_{1}(v)-f_{1}(v+i \gamma) .
\end{aligned}
$$

Now we can take the Trotter limit, $\lim _{N \rightarrow \infty} \ln \left[\phi_{+}(v) / \phi_{-}(v)\right]=i J \beta \sin \gamma \operatorname{coth} v$, with the result

$$
\begin{array}{r}
\ln \mathfrak{a}_{1}(v)=\beta\left(h_{3}-h_{1}\right)-\beta \frac{J \sinh ^{2} i \gamma}{\sinh v \sinh (v-i \gamma)} \\
\quad+\int_{\mathcal{C}} \bar{K}_{0}(v-w) \ln \left[1+\mathfrak{a}_{1}(w)\right] d w \\
\quad-\int_{\mathcal{C}} \bar{K}_{2}(v-w) \ln \left[1+\mathfrak{a}_{2}(w)\right] d w \\
\begin{aligned}
\ln \mathfrak{a}_{2}(v)=\beta\left(h_{2}-h_{1}\right)-\beta \frac{J \sinh ^{2} i \gamma}{\sinh v \sinh (v+i \gamma)} \\
+\int_{\mathcal{C}} \bar{K}_{1}(v-w) \ln \left[1+\mathfrak{a}_{1}(w)\right] d w
\end{aligned}
\end{array}
$$

where

$$
\begin{aligned}
\bar{K}_{0}(v) & =\frac{1}{2 \pi i} \frac{\sinh 2 i \gamma}{\sinh (v+i \gamma) \sinh (v-i \gamma)}, \\
\bar{K}_{1}(v) & =\frac{1}{2 \pi i} \frac{\sinh i \gamma}{\sinh (v) \sinh (v+i \gamma)}, \\
\bar{K}_{2}(v) & =\frac{1}{2 \pi i} \frac{\sinh i \gamma}{\sinh (v) \sinh (v-i \gamma)} .
\end{aligned}
$$

Eqs. (38) were derived assuming $\gamma \in(0, \pi / 2)$ and $v$ is outside the contour. For $v$ inside the contour we need to add a $\ln \left[1+\mathfrak{a}_{2}(v)\right]$ term on the right hand side of Eq. (38a) and a $\ln \left[1+\mathfrak{a}_{1}(v)\right]$ term on the right hand side of Eq. (38b). For $\gamma \in(\pi / 2, \pi)$ the same equations remain valid if we replace $\mathcal{C}$ with a similar rectangular contour with horizontal edges situated at $\pm i(\pi-\gamma-\epsilon) / 2$.

\section{B. Integral expression for the largest eigenvalue}

The largest eigenvalue of the QTM is analytic in a strip around the real axis, therefore it will be sufficient to derive an integral expression for $\ln \Lambda_{0}\left(v_{0}\right)$ with $v_{0}$ close to the real axis and then take the limit $v_{0} \rightarrow 0$ to obtain the free energy. For our purposes we choose $v_{0}=i u$ for which $\lambda_{3}\left(v_{0}\right)=0$ and ( $c$ is a constant)

$$
\Lambda_{0}\left(v_{0}\right)=\lambda_{1}\left(v_{0}\right)+\lambda_{2}\left(v_{0}\right)=c \frac{\phi_{+}\left(v_{0}\right) q_{1}^{(h)}\left(v_{0}\right)}{q_{2}\left(v_{0}\right)},
$$

where we have used the identity A1 and $q_{i}^{(h)}(v)$ are defined in Appendix A

Consider $v$ inside the contour $\mathcal{C}$. Then, inside the contour $\mathcal{C}^{\prime}$ depicted in Fig. 7, the function $1+\mathfrak{a}_{1}(v)$ has $N$ zeroes identified with the holes $\left\{v^{\prime}{ }_{j}{ }^{(1)}\right\}_{j=1}^{N}, N / 2$ poles located at $\left\{v_{j}^{(1)}-i \gamma\right\}_{j=1}^{N / 2}$ and $N / 2$ poles located at $\left\{v_{j}^{(2)}+i \gamma\right\}_{j=1}^{N / 2}$ (some of the holes and poles are modulo $i \pi)$. This means that around $\mathcal{C}^{\prime}$ the function $\ln \left[1+\mathfrak{a}_{1}(v)\right]$ has zero winding number. Using the identity A6 in the form $(d(v)=d \ln \sinh v / d v)$

$\int_{\mathcal{C}} d(v-w) \frac{\mathfrak{a}_{j}^{\prime}(w)}{1+\mathfrak{a}_{j}(w)} d w=-\int_{\mathcal{C}^{\prime}} d(v-w) \frac{\mathfrak{a}_{j}^{\prime}(w)}{1+\mathfrak{a}_{j}(w)} d w$

the right hand side can be computed as 36 with the result

$$
\begin{gathered}
\frac{1}{2 \pi i} \int_{\mathcal{C}} d(v-w) \frac{\mathfrak{a}_{1}^{\prime}(w)}{1+\mathfrak{a}_{1}(w)} d w=\sum_{j=1}^{N / 2} d\left(v-v_{j}^{(1)}+i \gamma\right) \\
+\sum_{j=1}^{N / 2} d\left(v-v_{j}^{(2)}-i \gamma\right)-\sum_{j=1}^{N} d\left(v-v_{j}^{(1)}\right) .
\end{gathered}
$$

After integration by parts with respect to $w$ and then integration with respect to $v$ we find

$$
\begin{gathered}
\frac{1}{2 \pi i} \int_{\mathcal{C}} d(v-w) \ln \left[1+\mathfrak{a}_{1}(w)\right] d w=-\ln q_{1}^{(h)}(v) \\
+\ln q_{1}(v+i \gamma)+\ln q_{2}(v-i \gamma)+c
\end{gathered}
$$

In a similar fashion using the fact that inside $\mathcal{C}^{\prime}$ the function $1+\mathfrak{a}_{2}(v)$ has $N / 2$ zeroes at the holes $\left\{v_{j}^{\prime(2)}\right\}_{j=1}^{N / 2}$ and $N / 2$ poles located at $\left\{v_{j}^{(1)}-i \gamma\right\}_{j=1}^{N / 2}$ (some modulo $i \pi$ ) we find

$$
\begin{aligned}
& \frac{1}{2 \pi i} \int_{\mathcal{C}} d(v-w) \ln [1+\left.\mathfrak{a}_{2}(w)\right] d w=-\ln q_{2}^{(h)}(v) \\
&+\ln q_{1}(v+i \gamma)+c .
\end{aligned}
$$

For $v$ inside $\mathcal{C}, v \pm i \gamma$ is outside of the contour. Therefore, from 36 we have

$$
\begin{array}{r}
\frac{1}{2 \pi i} \int_{\mathcal{C}} d(v-w) \ln \left[1+\mathfrak{a}_{1}(w)\right] d w=\ln q_{1}(v+i \gamma) \\
-\ln \phi_{-}(v+i \gamma)-\frac{N}{2} \sin \gamma \\
\frac{1}{2 \pi i} \int_{\mathcal{C}} d(v-w) \ln \left[1+\mathfrak{a}_{2}(w)\right] d w=\ln q_{2}(v-i \gamma)
\end{array}
$$




$$
-\ln \phi_{+}(v-i \gamma)-\frac{N}{2} \sin \gamma .
$$

Subtracting Eq. (44) from Eq.446a) and Eq. (45) from Eq. 46b we obtain

$$
\begin{gathered}
\int_{\mathcal{C}} \bar{K}_{1}(v-w) \ln \left[1+\mathfrak{a}_{1}(w)\right] d w=-\ln q_{1}^{(h)}(v) \\
+\ln q_{2}(v-i \gamma)+\ln \phi_{-}(v+i \gamma)+c \\
-\int_{\mathcal{C}} \bar{K}_{2}(v-w) \ln \left[1+\mathfrak{a}_{2}(w)\right] d w=-\ln q_{2}^{(h)}(v) \\
+\ln q_{1}(v+i \gamma)-\ln q_{2}(v-i \gamma) \\
+\ln \phi_{+}(v-i \gamma)+c .
\end{gathered}
$$

The importance of this result comes to light by noticing that the expression of the largest eigenvalue 42 can be rewritten using (A5) as

$$
\begin{array}{r}
\ln \Lambda_{0}\left(v_{0}\right)=\ln q_{1}^{(h)}\left(v_{0}\right)+\ln q_{2}^{(h)}\left(v_{0}\right) \\
\quad-\ln q_{1}\left(v_{0}+i \gamma\right)-\ln \left[1+\mathfrak{a}_{2}\left(v_{0}\right)\right]+c,
\end{array}
$$

and then using (47) as

$$
\begin{aligned}
& \ln \Lambda_{0}\left(v_{0}\right)=-\int_{\mathcal{C}} \bar{K}_{1}\left(v_{0}-w\right) \ln \left[1+\mathfrak{a}_{1}(w)\right] d w \\
& \quad+\int_{\mathcal{C}} \bar{K}_{2}\left(v_{0}-w\right) \ln \left[1+\mathfrak{a}_{2}(w)\right] d w-\ln \left[1+\mathfrak{a}_{2}\left(v_{0}\right)\right] \\
& \quad+\ln \left[\phi_{+}\left(v_{0}-i \gamma\right) \phi_{-}\left(v_{0}+i \gamma\right)\right]+c .
\end{aligned}
$$

The constant of integration can be computed by noticing that Eq. (48) is in fact valid for all $v$ in a narrow strip around the real axis with $\ln \left[\lambda_{1}(v)+\lambda_{2}(v)\right]$ replacing the left hand side. Considering the limit $v \rightarrow \infty$ and using $\lim _{v \rightarrow \infty}\left[\lambda_{1}(v)+\lambda_{2}(v)\right] /\left[\phi_{+}(v-i \gamma) \phi_{-}(v+i \gamma)\right]=e^{\beta h_{1}}+$ $e^{\beta h_{3}}$ we find

$$
c=\beta h_{1}+c^{\prime}, \quad \text { with } c^{\prime}=2 \ln \left[\left(1+e^{\beta\left(h_{3}-h_{1}\right)}\right)\left(1+e^{\beta\left(h_{2}-h_{1}\right)}\right)\right]
$$

Finally, by taking the Trotter limit, $N \rightarrow \infty$, and using $\lim _{N \rightarrow \infty} \ln \left[\phi_{+}\left(v_{0}-i \gamma\right) \phi_{-}\left(v_{0}+i \gamma\right)\right]=-J \cos \gamma \beta$ we obtain

$$
\begin{gathered}
\ln \Lambda_{0}(0)=c-J \beta \cos \gamma-\int_{\mathcal{C}} \bar{K}_{2}(w) \ln \left[1+\mathfrak{a}_{1}(w)\right] d w \\
\quad+\int_{\mathcal{C}} \bar{K}_{1}(w) \ln \left[1+\mathfrak{a}_{2}(w)\right] d w-\ln \left[1+\mathfrak{a}_{2}(0)\right] .
\end{gathered}
$$

This result was derived for $\gamma \in(0, \pi / 2)$ but it remains valid also for $\gamma \in(\pi / 2, \pi)$ if $\mathcal{C}$ is replaced by a rectangular contour with the horizontal edges situated at $\pm i(\pi-\gamma-$ $\epsilon) / 2$.

\section{Continuum limit}

The continuum limit (see Sec. VI) of the integral equations (38) and integral expression for the largest eigenvalue $(50)$ is the same as the one performed for the $2 \mathrm{CBG}$ and is presented in detail in [76. In the scaling limit we obtain Eq. (6) for the grandcanonical potential of the continuum model with the auxiliary functions satisfying the NLIEs (7).

\section{CONCLUSIONS}

In this paper we have derived an alternative thermodynamic description for the Bose-Fermi mixture in the QTM framework and performed a detailed analysis of the contact at zero and finite temperature. In the strong coupling regime the contact develops a pronounced local minimum as a function of the temperature which is accompanied by a significant momentum reconstruction at both low and large momenta. This momentum reconstruction can be experimentally detected and provides an identification of the transition from the TLL to the spinincoherent regime. In addition, we have also showed that the boundaries of the $\mathrm{QC}$ regions can be well mapped by the maxima of the grand canonical specific heat. Our results also hint at the possibility of deriving efficient thermodynamic descriptions for integrable $\kappa$-component $(\kappa>2)$, systems with contact interactions involving only $\kappa$ integral equations.

\section{ACKNOWLEDGMENTS}

O.I.P. acknowledges financial support from the LAPLAS 4 and 5 programs of the Romanian National Authority for Scientific Research (CNCS-UEFISCDI). Both authors are grateful to Deutsche Forschungsgemeinschaft (DFG) for support via Research Unit FOR 2316.

\section{Appendix A: Some useful identities}

In this Appendix we prove certain identities which are needed in the derivation of the integral expression of the largest QTM eigenvalue. First, we will prove that

$$
\lambda_{1}(v)+\lambda_{2}(v)=c \frac{\phi_{+}(v) q_{1}^{(h)}(v)}{q_{2}(v)},
$$

with $c$ a constant and $q_{1}^{(h)}(v)$ defined by

$$
q_{1}^{(h)}=\prod_{i=1}^{N} \sinh \left(v-{v_{i}^{\prime}}_{i}^{(1)}\right) .
$$

From the definition of the $\lambda_{j}(v)$ functions we obtain

$$
\lambda_{1}(v)+\lambda_{2}(v)=\frac{\phi_{+}(v) p_{1}(v)}{q_{1}(v) q_{2}(v)},
$$

with $p_{1}(v)=\left(\phi_{-}(v+i \gamma) q_{1}(v-i \gamma) q_{2}(v) e^{\beta h_{3}}+\phi_{-}(v) q_{1}(v+\right.$ $\left.i \gamma) q_{2}(v-i \gamma) e^{\beta h_{1}}\right)$. The equation $p_{1}(v)=0$ (which is equivalent to $\mathfrak{a}_{1}(v)=-1$ ) has $3 N / 2$ solutions which are the $N / 2$ Bethe roots, $\left\{v_{j}^{(1)}\right\}_{j=1}^{N / 2}$, and the $N$ holes $\left\{v^{\prime(1)}\right\}_{j=1}^{N}$. Also $p_{1}(v+i \pi)=(-1)^{3 N / 2} p(v)$ and $\lim _{v \rightarrow \infty} p_{1}(v) / \sinh ^{3 N / 2} v=$ const. which shows that $p_{1}(v)=c q_{1}(v) q_{1}^{(h)}(v)$. This concludes the proof of A1. 
A similar identity is

$$
\lambda_{2}(v)+\lambda_{3}(v)=c \frac{\phi_{-}(v) q_{2}(v-i \gamma) q_{2}^{(h)}(v)}{q_{1}(v)}
$$

with

$$
q_{2}^{(h)}=\prod_{i=1}^{N / 2} \sinh \left(v-v_{i}^{\prime(2)}\right) .
$$

Again, from the definition we have

$$
\lambda_{2}(v)+\lambda_{3}(v)=\frac{\phi_{-}(v) q_{2}(v-i \gamma) p_{2}(v)}{q_{1}(v) q_{2}(v)}
$$

$\left\{v_{j}^{(2)}\right\}_{j=1}^{N / 2}$, and the $N / 2$ holes $\left\{v_{j}^{\prime(2)}\right\}_{j=1}^{N / 2}$. In addition we have $p_{2}(v+i \pi)=(-1)^{N} p_{2}(v)$ and $\lim _{v \rightarrow \infty} p_{2}(v) / \sinh ^{N} v=$ const. which shows that $p_{2}(v)$ can be written as $p_{2}(v)=c q_{2}(v) q_{2}^{(h)}(v)$, concluding the proof of A3. Also, we have $\ln \left[1+\mathfrak{a}_{2}(v)\right]=\ln \left(p_{2}(v) / \phi_{+}(v) q_{1}(v+i \gamma)\right.$ which is equivalent to

$$
\begin{aligned}
& -\ln \phi_{+}(v)+\ln q_{2}(v)-\ln q_{1}(v+i \gamma) \\
& \quad+\ln q_{2}^{(h)}(v)-\ln \left[1+\mathfrak{a}_{2}(v)\right]+\text { const. }=0 .
\end{aligned}
$$

In Sec. VII B we will also use $\left(d(v)=\frac{d}{d v} \ln \sinh v\right)$

$$
\int_{\mathcal{C}+\mathcal{C}^{\prime}} d(v-w) \frac{\mathfrak{a}_{j}^{\prime}(w)}{1+\mathfrak{a}_{j}(w)} d w=0,
$$

with $p_{2}(v)=\left(\phi_{+}(v) q_{1}(v+i \gamma) e^{\beta h_{1}}+q_{1}(v) \phi_{+}(v+i \gamma) e^{\beta h_{2}}\right)$. with the contours depicted in Fig. 7. The proof is similar The equation $p_{2}(v)=0$ (equivalent to $\mathfrak{a}_{2}(v)=-1$ ) has $N$ solutions which are the $N / 2$ Bethe roots, with the one described in [76] and [77] for the $2 \mathrm{CBG}$ and $2 \mathrm{CFG}$ cases and is left to the reader.
[1] I. Bloch, J. Dalibard, and W. Zwerger, Many-body physics with ultracold gases, Rev. Mod. Phys. 80, 885 (2008).

[2] M.A. Cazalilla, R. Citro, T. Giamarchi, E. Orignac, and M. Rigol, One dimensional bosons: From condensed matter systems to ultracold gases, Rev. Mod. Phys. 83, 1405 (2011)

[3] X.-W. Guan, M.T. Batchelor, and C. Lee, Fermi gases in one dimension: From Bethe ansatz to experiments, Rev. Mod. Phys. 85, 1633 (2013).

[4] R. Onofrio, Cooling and thermometry of atomic Fermi gases, Phys.-Usp. 59, 1129 (2016).

[5] K.K. Das, Bose-Fermi Mixtures in One Dimension, Phys. Rev. Lett. 90, 170403 (2003).

[6] Z. Akdeniz, P. Vignolo, and M.P. Tosi, Boson-fermion mixtures inside an elongated cigar-shaped trap, J. Phys. B 38, 2933 (2005).

[7] F.M. Marchetti, Th. Jolicoeur, and M.M. Parish, Stability and Pairing in Quasi-One-Dimensional Bose-Fermi Mixtures, Phys. Rev. Lett. 103, 105304 (2009).

[8] D. Rakshit, T. Karpiuk, M. Brewczyk, M. Lewenstein, and M. Gajda, Self-bound Bose-Fermi liquids in lower dimensions, arXiv:1808.04793.

[9] M.A. Cazalilla and A. F. Ho, Instabilities in Binary Mixtures of One-Dimensional Quantum Degenerate Gases, Phys. Rev. Lett. 91, 150403 (2003).

[10] L. Mathey, D.-W. Wang, W. Hofstetter, M. D. Lukin, and E. Demler, Luttinger Liquid of Polarons in OneDimensional Boson-Fermion Mixtures, Phys. Rev. Lett. 93, 120404 (2004).

[11] H. Frahm and G. Palacios, Correlation functions of onedimensional Bose-Fermi mixtures, Phys. Rev. A 72, 061604(R) (2005).

[12] L. Mathey and D.-W. Wang, Phase diagrams of onedimensional Bose-Fermi mixtures of ultracold atoms, Phys. Rev. A 75, 013612 (2007).

[13] E. Orignac, M. Tsuchiizu, and Y. Suzumura, Com- petition of superfluidity and density waves in onedimensional Bose-Fermi mixtures, Phys. Rev. A 81, 053626 (2010).

[14] B. Reichert, A. Petković, and Z. Ristivojevic, Quasiparticle decay in a one-dimensional Bose-Fermi mixture, Phys. Rev. B 95, 045426 (2017).

[15] P. Schlottmann, Threshold singularities in the onedimensional supersymmetric boson-fermion gas mixture, Int. J. Mod. Phys. B 32, 1850221 (2018).

[16] T. Miyakawa, H. Yabu, and T. Suzuki, Peierls instability, periodic Bose-Einstein condensates, and density waves in quasi-one-dimensional boson-fermion mixtures of atomic gases, Phys. Rev. A 70, 013612 (2004).

[17] E. Nakano and H. Yabu, Density waves in a quasione-dimensional atomic gas mixture of bosons and twocomponent fermions, Phys. Rev. A 72, 043602 (2005).

[18] C.K. Lai and C.N. Yang, Ground-State Energy of a Mixture of Fermions and Bosons in One Dimension with a Repulsive $\delta$-Function Interaction, Phys. Rev. A 3, 393 (1971).

[19] C.K. Lai, Thermodynamics of Fermions in One Dimension with a $\delta$-Function Interaction, Phys. Rev. Lett. 26, 1472 (1971).

[20] C.K. Lai, Thermodynamics of a mixture of fermions and bosons in one dimension with a repulsive $\delta$ function potential, J. Math. Phys. 15, 954 (1974).

[21] M.T. Batchelor, M. Bortz, X.-W. Guan, and N. Oelkers, Exact results for the one-dimensional mixed bosonfermion interacting gas, Phys. Rev. A 72, 061603(R) (2005).

[22] N. Oelkers, M.T. Batchelor, M. Bortz, and X.W. Guan, Bethe Ansatz study of one-dimensional Bose and Fermi gases with periodic and hard wall boundary conditions, J. Phys. A 39, 1073 (2006).

[23] X.-W. Guan, M.T. Batchelor, and J.-Y. Lee, Magnetic ordering and quantum statistical effects in strongly repulsive Fermi-Fermi and Bose-Fermi mixtures, Phys. 
Rev. A 78, 023621 (2008).

[24] A. Imambekov and E. Demler, Exactly solvable case of a one-dimensional BoseFermi mixture, Phys. Rev. A 73, 021602(R) (2006).

[25] A. Imambekov and E. Demler, Applications of exact solution for strongly interacting one-dimensional BoseFermi mixture: Low-temperature correlation functions, density profiles, and collective modes, Ann. Phys. 321, 2390 (2006).

[26] Z.-X. Hu, Q.-L. Zhang and Y.-Q. Li Ground state properties of one-dimensional Bose-Fermi mixtures, J. Phys. A 39, 351 (2006).

[27] X. Yin, S. Chen, and Y. Zhang, Yang-Yang thermodynamics of a Bose-Fermi mixture, Phys. Rev. A 79, 053604 (2009).

[28] S.-J. Gu, J. Cao, S. Chen, and H.-Q. Lin, Quantum phase transition and elementary excitations of a Bose-Fermi mixture in a one-dimensional optical lattice, Phys. Rev. B 80, 224508 (2009).

[29] Hao Y.-J., Ground State Density Distribution of BoseFermi Mixture in a One-Dimensional Harmonic Trap, Chinese Phys. Lett. 28, 010302 (2011).

[30] Hao Y.-J., Composite-fermionization of the mixture composed of Tonks gas and Fermi gas, Chinese Phys. B 20, 060307 (2011).

[31] X. Yin, X.-W. Guan, Y. Zhang, and S. Chen Quantum criticality of a one-dimensional Bose-Fermi mixture, Phys. Rev. A 85, 013608 (2012).

[32] P. Schlottmann, Mixture of interacting supersymmetric spinless fermions and bosons in a one-dimensional trap, Mod. Phys. Lett. B, 30, 1630007 (2016).

[33] Y. Takeuchi and H. Mori, Mixing-demixing transition in one-dimensional boson-fermion mixtures, Phys. Rev. A 72, 063617 (2005).

[34] L. Pollet, M. Troyer, K. Van Houcke, and S.M.A. Rombouts, Phase Diagram of Bose-Fermi Mixtures in One-Dimensional Optical Lattices, Phys. Rev. Lett. 96, 190402 (2006).

[35] P. Sengupta and L.P. Pryadko, Quantum degenerate Bose-Fermi mixtures on one-dimensional optical lattices, Phys. Rev. B 75, 132507 (2007).

[36] A. Mering and M. Fleischhauer, One-dimensional BoseFermi-Hubbard model in the heavy-fermion limit, Phys. Rev. A 77, 023601 (2008).

[37] A. Zujev, A. Baldwin, R.T. Scalettar, V.G. Rousseau, P.J.H. Denteneer, and M. Rigol, Superfluid and Mottinsulator phases of one-dimensional Bose-Fermi mixtures, Phys. Rev. A 78, 033619 (2008).

[38] X. Barillier-Pertuisel, S. Pittel, L. Pollet, and P. Schuck, Boson-fermion pairing in Bose-Fermi mixtures on onedimensional optical lattices, Phys. Rev. A 77, 012115 (2008).

[39] M. Rizzi and A. Imambekov, Pairing of onedimensional Bose-Fermi mixtures with unequal masses, Phys. Rev. A 77, 023621 (2008).

[40] H. Wang, Y. Hao, and Y. Zhang, Density-functional theory for one-dimensional harmonically trapped BoseFermi mixture, Phys. Rev. A 85, 053630 (2012).

[41] K.K. Nielsen, Z. Wu, and G.M. Bruun, Higher first Chern numbers in one-dimensional Bose-Fermi mixtures, New J. Phys. 20, 025005 (2018).

[42] M.D. Girardeau and A. Minguzzi, Soluble Models of Strongly Interacting Ultracold Gas Mixtures in Tight Waveguides, Phys. Rev. Lett. 99, 230402 (2007).
[43] B. Fang, P. Vignolo, C. Miniatura, and A. Minguzzi, Fermionization of a strongly interacting Bose-Fermi mixture in a one-dimensional harmonic trap, Phys. Rev. A 79, 023623 (2009).

[44] B. Fang, P. Vignolo, M. Gattobigio, C. Miniatura, and A. Minguzzi, Exact solution for the degenerate groundstate manifold of a strongly interacting one-dimensional Bose-Fermi mixture, Phys. Rev. A 84, 023626 (2011).

[45] J. Decamp, J. Jünemann, M. Albert, M. Rizzi, A. Minguzzi, P. Vignolo, High-momentum tails as magnetic structure probes for strongly-correlated $S U(\kappa)$ fermionic mixtures in one-dimensional traps, Phys. Rev. A 94, 053614 (2016).

[46] J. Decamp, J. Jünemann, M. Albert, M. Rizzi, A. Minguzzi, P. Vignolo, Strongly correlated one-dimensional Bose-Fermi quantum mixtures: symmetry and correlations, New J. Phys. 19, 125001 (2017).

[47] J. Decamp, P. Armagnat, B. Fang, M. Albert, A. Minguzzi, and P. Vignolo, Exact density profiles and symmetry classification for strongly interacting multicomponent Fermi gases in tight waveguides, New J. Phys., 18, 055011 (2016).

[48] K. Lelas, D. Jukić, and H. Buljan, Ground-state properties of a one-dimensional strongly interacting BoseFermi mixture in a double-well potential, Phys. Rev. A 80, 053617 (2009).

[49] X. Lü, X. Yin, and Y. Zhang, Hard-core Bose-Fermi mixture in one-dimensional split traps, Phys. Rev. A 81, 043607 (2010).

[50] S. Chen, J. Cao, and S.-J. Gu, Mixture of TonksGirardeau gas and Fermi gas in one-dimensional optical lattices, Phys. Rev. A 82, 053625 (2010).

[51] H. Hu, L. Guan, and S. Chen, Strongly interacting BoseFermi mixtures in one dimension, New J. Phys. 18, 025009 (2016).

[52] F. Deuretzbacher, D. Becker, J. Bjerlin, S. M. Reimann, and L. Santos, Spin-chain model for strongly interacting one-dimensional Bose-Fermi mixtures, Phys. Rev. A 95, 043630 (2017).

[53] F. Deuretzbacher, K. Fredenhagen, D. Becker, K. Bongs, K. Sengstock, and D. Pfannkuche, Exact Solution of Strongly Interacting Quasi-One-Dimensional Spinor Bose Gases, Phys. Rev. Lett. 100, 160405 (2008).

[54] F. Deuretzbacher, D. Becker, J. Bjerlin, S. M. Reimann, and L. Santos, Quantum magnetism without lattices in strongly interacting one-dimensional spinor gases, Phys. Rev. A 90, 013611 (2014).

[55] A.G. Volosniev, D.V. Fedorov, A.S. Jensen, M. Valiente, and N.T. Zinner, Strongly interacting confined quantum systems in one dimension, Nat. Commun. 5, 5300 (2014).

[56] N.J.S. Loft, L.B. Kristensen, A.E. Thomsen, and N.T. Zinner, Comparing models for the ground state energy of a trapped one-dimensional Fermi gas with a single impurity, J. Phys. B 49, 125305 (2016).

[57] A.S. Dehkharghani, F.F. Bellotti, and N.T. Zinner, Analytical and numerical studies of Bose-Fermi mixtures in a one-dimensional harmonic trap, J. Phys. B 50, 144002 (2017)

[58] F.F. Bellotti, A.S. Dehkharghani, and N.T. Zinner, Comparing numerical and analytical approaches to strongly interacting two-component mixtures in one dimensional traps, Eur. Phys. J. D 71, 37 (2017). 
[59] R.E. Barfknecht, I. Brouzos, and A. Foerster, Contact and static structure factor for bosonic and fermionic mixtures, Phys. Rev. A 91, 043640 (2015).

[60] J. Chen, J. M. Schurer, and P. Schmelcher, Bunchingantibunching crossover in harmonically trapped few-body Bose-Fermi mixtures, Phys. Rev. A 98, 023602 (2018).

[61] P. Siegl, S. I. Mistakidis, and P. Schmelcher, ManyBody Expansion Dynamics of a Bose-Fermi Mixture Confined in an Optical Lattice, arXiv:1803.07881.

[62] S. Sachdev, Quantum Phase Transitions, (Cambridge University Press, Cambridge, UK, 2011).

[63] V.E. Korepin, N.M. Bogoliubov, and A.G. Izergin, Quantum Inverse Scattering Method and Correlation Functions, (Cambridge University Press, Cambridge, UK, 1993).

[64] F.H.L. Essler, H. Frahm, F. Göhmann, A. Klümper, V. E. Korepin, The One-Dimensional Hubbard Model, (Cambridge University Press, Cambridge, UK, 2005).

[65] C.N. Yang and C.P. Yang, Thermodynamics of a OneDimensional System of Bosons with Repulsive DeltaFunction Interaction, J. Math. Phys. 10, 1115 (1969).

[66] M. Takahashi, Thermodynamics of One-Dimensional Solvable Models, (Cambridge University Press, Cambridge, UK, 1999).

[67] C.N. Yang, Some Exact Results for the Many-Body Problem in one Dimension with Repulsive DeltaFunction Interaction, Phys. Rev. Lett. 19, 1312 (1967).

[68] M. Gaudin, Un systeme a une dimension de fermions en interaction, Phys. Lett. A 24, 55 (1967).

[69] M. Suzuki, Transfer-matrix method and Monte Carlo simulation in quantum spin systems, Phys. Rev. B 31, 2957 (1985).

[70] M. Suzuki and M. Inoue, The ST-Transformation Approach to Analytic Solutions of Quantum Systems. I: General Formulations and Basic Limit Theorems, Prog. Theor. Phys. 78, 787 (1987).

[71] T. Koma, Thermal Bethe-Ansatz Method for the OneDimensional Heisenberg Model, Prog. Theor. Phys. 78, 1213 (1987)

[72] J. Suzuki, Y. Akutsu, and M. Wadati, A New Approach to Quantum Spin Chains at Finite Temperature, J. Phys. Soc. Jpn. 59, 2667 (1990).

[73] A. Klümper, Free energy and correlation lengths of quantum chains related to restricted solid-on-solid lattice models, Ann. Phys. 504, 540 (1992).

[74] A. Klümper, Thermodynamics of the anisotropic spin1/2 Heisenberg chain and related quantum chains, Z. Phys. B: Condens. Matter 91, 507 (1993).

[75] A. Klümper and O.I. Pâţu, Efficient thermodynamic description of multicomponent one-dimensional Bose gases, Phys. Rev. A 84, 051604(R)(2011).

[76] O.I. Pâţu and A. Klümper, Thermodynamics, density profiles and correlation functions of the inhomogeneous one-dimensional spinor Bose gas, Phys. Rev. A 92, 043631 (2015).

[77] O.I. Pâţu and A. Klümper, Thermodynamics, contact, and density profiles of the repulsive Gaudin-Yang model, Phys. Rev. A 93, 033616 (2016).

[78] S. Tan, Energetics of a strongly correlated Fermi gas, Ann. Phys. 323, 2952 (2008).

[79] S. Tan, Large momentum part of a strongly correlated Fermi gas, Ann. Phys. 323, 2971 (2008).

[80] S. Tan, Generalized virial theorem and pressure relation for a strongly correlated Fermi gas, Ann. Phys. 323,
2987 (2008)

[81] M. Olshanii and V. Dunjko, Short-Distance Correlation Properties of the Lieb-Liniger System and Momentum Distributions of Trapped One-Dimensional Atomic Gases, Phys. Rev. Lett. 91, 090401 (2003).

[82] E. Braaten and L. Platter, Exact Relations for a Strongly-interacting Fermi Gas from the Operator Product Expansion, Phys. Rev. Lett. 100, 205301 (2008).

[83] E. Braaten, D. Kang, and L. Platter, Exact Relations for a Strongly-interacting Fermi Gas near a Feshbach Resonance, Phys. Rev. A 78, 053606 (2008).

[84] S. Zhang and A.J. Leggett, Universal properties of the ultracold Fermi gas, Phys. Rev. A 79, 023601 (2009).

[85] R. Combescot, F. Alzetto, and X. Leyronas, Particle distribution tail and related energy formula, Phys. Rev. A 79, 053640 (2009).

[86] F. Werner and Y. Castin, General relations for quantum gases in two and three dimensions: Two-component fermions, Phys. Rev. A 86, 013626 (2012).

[87] F. Werner and Y. Castin, General relations for quantum gases in two and three dimensions. II. Bosons and mixtures, Phys. Rev. A 86053633 (2012).

[88] M. Valiente, N.T. Zinner, and K. Mølmer, Universal relations for the two-dimensional spin-1/2 Fermi gas with contact interactions, Phys. Rev. A 84, 063626 (2011).

[89] M. Valiente, N.T. Zinner, and K. Mølmer, Universal properties of Fermi gases in arbitrary dimensions, Phys. Rev. A 86, 043616 (2012).

[90] M. Barth and W. Zwerger, Tan relations in one dimension, Ann. Phys. 326, 2544 (2011).

[91] O.I. Pâţu and A. Klümper, Universal Tan relations for quantum gases in one dimension, Phys. Rev. A 96, 063612 (2017).

[92] O.I. Pâţu, A. Klümper, and A. Foerster, Universality and quantum criticality of the one-dimensional spinor Bose gas, Phys. Rev. Lett. 120, 243402 (2018).

[93] E.H. Lieb and W. Liniger, Exact Analysis of an Interacting Bose Gas. I. The General Solution and the Ground State, Phys. Rev. 130, 1605 (1963).

[94] M. Takahashi, On the Validity of Collective Variable Description of Bose Systems, Progr. Theor. Phys. 53, 386 (1975).

[95] C.A. Tracy and H. Widom, On the ground state energy of the delta-function Bose gas, J. Phys. A 49, 294001 (2016).

[96] S. Prolhac, Ground state energy of the $\delta$-Bose and Fermi gas at weak coupling from double extrapolation, J. Phys. A 50, 144001 (2017).

[97] G. Lang, F. Hekking, and A. Minguzzi, Ground-state energy and excitation spectrum of the Lieb-Liniger model: accurate analytical results and conjectures about the exact solution, SciPost Phys. 3, 003 (2017).

[98] V.V. Cheianov, H. Smith, and M.B. Zvonarev, Lowtemperature crossover in the momentum distribution of cold atomic gases in one dimension, Phys. Rev. A 71, 033610 (2005).

[99] A. Berkovich A and J.H. Lowenstein, Correlation function of the one-dimensional Fermi gas in the infinitecoupling limit (repulsive case), Nucl. Phys. B 285, 70 (1987).

[100] A. Berkovich, Temperature and magnetic fielddependent correlators of the exactly integrable $(1+1)$ dimensional gas of impenetrable fermions, J. Phys. A 241543 (1991). 
[101] V.V. Cheianov and M.B. Zvonarev, Nonunitary SpinCharge Separation in a One-Dimensional Fermion Gas, Phys. Rev. Lett. 92, 176401 (2004).

[102] G.A. Fiete and L. Balents, Green's Function for Magnetically Incoherent Interacting Electrons in One Dimension, Phys. Rev. Lett. 93, 226401 (2004).

[103] G.A. Fiete, Colloquium: The spin-incoherent Luttinger liquid, Rev. Mod. Phys. 79, 801 (2007).

[104] Q. Zhou and T.-L. Ho, Signature of Quantum Criticality in the Density Profiles of Cold Atom Systems, Phys. Rev. Lett. 105, 245702 (2010).

[105] F. He, Y. Jiang, Y.-C. Yu, H.-Q. Lin, and X.-W. Guan, Quantum criticality of spinons, Phys. Rev. B 96, 220401(R) (2017).

[106] B. Yang, Y.-Y. Chen, Y.-G. Zheng, H. Sun, H.-N. Dai, X.-W. Guan, Z.-S. Yuan, and J.-W. Pan, Quantum criticality and the Tomonaga-Luttinger liquid in onedimensional Bose gases, Phys. Rev. Lett. 119, 165701 (2017).

[107] O. Breunig, M. Garst, A. Klümper, J. Rohrkamp, M.M. Turnbull, T. Lorenz, Quantum criticality in the spin1/2 Heisenberg chain system copper pyrazine dinitrate, Science Advances 3, eaao3773 (2017).

[108] Y.-C. Yu, Y.-Y. Chen, H.-Q. Lin, R.A. Römer, and X.-W. Guan, Dimensionless ratios: Characteristics of quantum liquids and their phase transitions, Phys. Rev. B 94, 195129 (2016).

[109] X.-W. Guan, X.-G. Yin, A. Foerster, M.T. Batchelor, C.-H. Lee, and H.-Q. Lin, Wilson Ratio of Fermi Gases in One Dimension, Phys. Rev. Lett. 111, 130401 (2013).

[110] Y. Maeda, C. Hotta, and M. Oshikawa, Universal Temperature Dependence of the Magnetization of Gapped Spin Chains, Phys. Rev. Lett. 99, 057205 (2007).

[111] K. Ninios, T. Hong, T. Manabe, C. Hotta, S. N. Herringer, M. M. Turnbull, C.P. Landee, Y. Takano, and H.B. Chan, Wilson Ratio of a Tomonaga-Luttinger Liquid in a Spin- $\frac{1}{2}$ Heisenberg Ladder, Phys. Rev. Lett. 108, 097201 (2012).

[112] S. Sachdev, T. Senthil, and R. Shankar, Finitetemperature properties of quantum antiferromagnets in a uniform magnetic field in one and two dimensions, Phys. Rev. B 50, 258 (1994).

[113] J. Y. Lee, X. W. Guan, K. Sakai, and M. T. Batch- elor, Thermodynamics, spin-charge separation, and correlation functions of spin-1/2 fermions with repulsive interaction, Phys. Rev. B 85, 085414 (2012).

[114] J.H.H. Perk and C. L. Schultz, New families of commuting transfer matrices in q-state vertex models, Phys. Lett. A 84, 407 (1981).

[115] C.L. Schultz, Eigenvectors of the multi-component generalization of the six-vertex model, Phys. A 122, 71 (1983).

[116] O. Babelon, H. J. de Vega, and C.-M. Viallet, Exact solution of the $Z_{n+1} \times Z_{n+1}$ symmetric generalization of the XXZ model, Nucl. Phys. B 200, 266 (1982).

[117] H.J. de Vega, Yang-Baxter algebras, integrable theories and quantum groups, Int. J. Mod. Phys. A 4, 2371 (1989).

[118] H.J. de Vega and E. Lopes, Exact solution of the PerkSchultz model, Phys. Rev. Lett. 67, 489 (1991).

[119] E. Lopes, Exact solution of the multi-component generalized six-vertex model, Nucl. Phys. B 370, 636 (1992).

[120] A. Seel, T. Bhattacharyya, F. Göhmann, A. Klümper, A note on the spin-1/2 XXZ chain concerning its relation to the Bose gas, J. Stat. Mech. P08030 (2007).

[121] B. Pozsgay, Local correlations in the 1D Bose gas from a scaling limit of the XXZ chain, J. Stat. Mech. P11017 (2011).

[122] O.I. Pâţu and A Klümper, Correlation lengths of the repulsive one-dimensional Bose gas, Phys. Rev. A 88, 033623 (2013).

[123] E. T. Whittaker and G. N. Watson, A Course of Modern Analysis, (Cambridge University Press 1927).

[124] F. Göhmann, Algebraic Bethe ansatz for the gl(1|2) generalized model and Lieb-Wu equations, Nucl. Phys. B 620, 501 (2002).

[125] F. Göhmann and A. Seel, Algebraic Bethe ansatz for the gl(1|2) generalized model: II. The three gradings, J. Phys. A 37, 2843 (2004).

[126] D. Arnaudon, N. Crampe, A. Doikou, L. Frappat, and E. Ragoucy, Spectrum and Bethe Ansatz Equations for the $\mathcal{U}_{q}(g l(\mathcal{N}))$ Closed and Open Spin Chains in any Representation, Ann. Inst. Henri Poincare 7, 1217 (2006).

[127] S. Belliard and E. Ragoucy, The nested Bethe ansatz for 'all' closed spin chains, J. Phys. A 41, 295202 (2008). 